\title{
Repetitive Dosing of Fumed Silica Leads to Pro-Fibrogenic Effects Through Unique Structure-Activity Relationships and Biopersistence in the Lung
}

\author{
Bingbing Sun ${ }^{1}$, Xiang Wang ${ }^{2}$, Yu-Pei Liao ${ }^{1}$, Zhaoxia $\mathrm{Ji}^{2}$, Chong Hyun Chang ${ }^{2}$, Suman \\ Pokhrel $^{3}$, Justine $\mathrm{Ku}^{4}$, Xiangsheng Liu ${ }^{1}$, Meiying Wang ${ }^{1}$, Darren R. Dunphy ${ }^{5}$, Ruibin $\mathrm{Li}^{1,6}$, \\ Huan Meng ${ }^{1}$, Lutz Mädler ${ }^{3}$, C. Jeffrey Brinker ${ }^{5,7,8}$, André E. Nel ${ }^{1,2, *}$, and Tian Xia ${ }^{1,2, *}$ \\ ${ }^{1}$ Division of NanoMedicine, Department of Medicine, University of California, Los Angeles, CA \\ 90095, United States \\ ${ }^{2}$ California NanoSystems Institute, University of California, Los Angeles, CA 90095, United States \\ ${ }^{3}$ Foundation Institute of Materials Science (IWT), Department of Production Engineering, \\ University of Bremen, Germany \\ ${ }^{4}$ Department of Ecology and Evolutionary Biology, University of California, Los Angeles, CA \\ 90095, United States \\ ${ }^{5}$ Department of Chemical and Nuclear Engineering, University of New Mexico, Albuquerque, New \\ Mexico 87131, United States \\ ${ }^{6}$ School for Radiological and Interdisciplinary Sciences (RAD-X), Collaborative Innovation Center \\ of Radiation Medicine of Jiangsu Higher Education Institutions, Soochow University, Suzhou \\ 215123, China \\ ${ }^{7}$ Department of Molecular Genetics and Microbiology, University of New Mexico, Albuquerque, \\ New Mexico 87131, United States \\ ${ }^{8}$ Self-Assembled Materials Department, Sandia National Laboratories, PO Box 5800 MS1349, \\ Albuquerque, New Mexico 87185, United States
}

\begin{abstract}
Contrary to the notion that the use of fumed silica in consumer products can "generally (be) regarded as safe" (GRAS), the high surface reactivity of pyrogenic silica differs from other forms of synthetic amorphous silica (SAS), including the capacity to induce acute membrane damage and acute pro-inflammatory changes in the murine lung. In addition, the chain-like structure and reactive surface silanols also allow fumed silica to activate the NLRP3 inflammasome, leading to IL-1 $\beta$ production. This pathway is known to be associated with sub-chronic inflammation and pro-
\end{abstract}

\footnotetext{
*Address correspondence to txia@ucla.edu or anel@mednet.ucla.edu.

Supporting Information

The Supporting Information is available free of charge on the ACS Publications website at DOI: XXX.

Table of dosimetry calculations for fumed silica; figure of fumed silica dissolution in simulated biological fluids; figure of the induction of IL-1 $\beta$ production by fumed silica was reduced by Ti-doping; figure of comparative analysis of the cytokine kinetics of single $v s$. repetitive dose fumed silica exposures; figure of comparative analysis of lung inflammation kinetics induced by single $v s$. repetitive fumed silica exposures; figure of Ti doping ameliorated the pro-fibrogenic effects of fumed silica during repetitive dosing.
} 
fibrogenic effects in the lung by a-quartz and carbon nanotubes. However, different from the latter materials, bolus dose instillation of $21 \mathrm{mg} / \mathrm{kg}$ fumed silica did not induce sustained IL-1 $\beta$ production or sub-chronic pulmonary effects. In contrast, the NLRP3 inflammasome pathway was continuously activated by repetitive dose administration of $3 \times 7 \mathrm{mg} / \mathrm{kg}$ fumed silica, one week apart. We also found that while single-dose exposure failed to induce pro-fibrotic effects in the lung, repetitive dosing can trigger increased collagen production, even at $3 \times 3 \mathrm{mg} / \mathrm{kg}$. The change between bolus and repetitive dosing was due to a change in lung clearance, with recurrent dosing leading to fumed silica biopersistence, sustained macrophage recruitment and activation of the NLRP3 pathway. These sub-chronic pro-inflammatory effects disappeared when less surface reactive titanium-doped fumed silica was used for recurrent administration. All considered, these data indicate that while fumed silica may be regarded as safe for some applications, we should reconsider the GRAS label during repetitive or chronic inhalation exposure conditions.

\section{TOC}

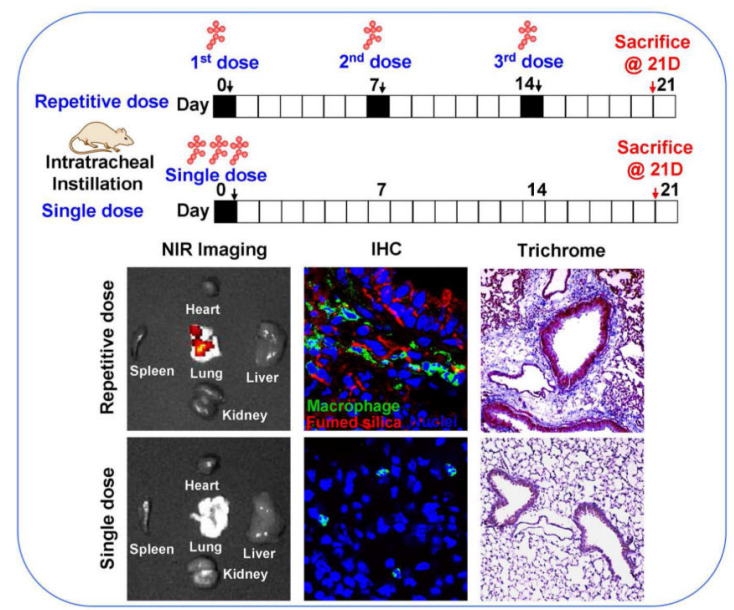

\section{Keywords}

fumed silica; metal doping; lung fibrosis; biopersistence; dissolution

The fumed silica market is the third largest aerosol commodity in the industrial sector by trade value and the fourth largest by volume. ${ }^{1}$ Moreover, the demand for these products are continuing to increase due to their expanded use in foods, pharmaceuticals, cosmetics, printer toners, tires and other industrial applications. ${ }^{2}$ Synthetic amorphous silica (SAS), in the form of pyrogenic (fumed), precipitated, gel or colloidal silica is "generally regarded as safe" (GRAS) by the Food and Drug Administrations (FDA). ${ }^{3}$ However, we have recently demonstrated that if different types of SAS are compared on the basis of structure-activity relationships (SARs), fumed silica exhibits a unique set of physicochemical properties that may distinguish itself from other types of SAS in terms of product safety. ${ }^{4}$ Fumed or pyrogenic silica is synthesized at high temperature and then thermally quenched to result in structures that differ from colloidal or precipitated silica. Fumes silica is characterized by chain-like aggregate morphologies and the presence of highly energetic three-membered siloxane rings, which can deconstruct under aqueous biological conditions to display highly 
reactive, H-bonded surface silanol groups. ${ }^{4}$ Others and we have demonstrated that these reactive silanols can lead to cell membrane damage, red cell lysis, and the initiation of acute inflammatory effects in cells and the lung. ${ }^{2,4-6}$ However, it is generally agreed upon that the acute pulmonary effects of SAS are reversible and not associated with permanent lung damage. Changing the particles surface silanol density by hydration/calcination or doping with titanium or aluminum generates a gradient of pro-inflammatory effects in the lung. ${ }^{2}$ In fact, fumed silica doping could be regarded as a safer design feature for acute lung injury. ${ }^{2}$

A key question now becomes whether the reactive fumed silica surface could pose chronic pulmonary hazard during repetitive exposure, e.g., within the workplace? While workers exposed to precipitated or fumed silica do not develop a known pneumoconiosis, pulmonary fibrosis has been reported in employees exposed to amorphous silica dust during silicon metal production. ${ }^{7}$ It is also known that diatomaceous earth workers can develop pneumoconiosis. ${ }^{8}$ Although there are a number of experimental animal studies in the literature showing the potential for SAS (colloidal and precipitated silica) to induce lung fibrosis, ${ }^{9}, 10$ surprisingly few studies have directly addressed this outcome for fumed silica. ${ }^{11}$ The most comprehensive is a 12 month exposure study in rabbits demonstrating that fumed silica can induce focal cellular infiltration and perialveolar collagen deposition in the lung. ${ }^{12}$ The general consensus appears to be that while the sub-chronic hazard potential of SAS is modest and reversible, repetitive exposures may introduce long term pulmonary hazard. ${ }^{13,14}$ However, it is not clear whether the possibility of more persistent lung injury by fumed silica has been sufficiently investigated in light of its in vitro and acute lung toxicological potential in the lung. One could also question whether a GRAS designation for fumed silica is appropriate?

In addition to the expression of three-membered siloxane rings and highly reactive silanols that could contribute to hazard, it is also important to consider the solubility and biopersistence of fumed silica when contemplating its longer term effects in the lung. Biopersistence is an important characteristic of a-quartz, a form of crystalline silica that could lead to silicosis in mineworkers. ${ }^{15}$ Compared to the low degree of a-quartz solubility, it is known that pyrogenic, precipitated and colloidal silica are soluble SAS materials in simulated biological fluids, with a size- and $\mathrm{pH}$-dependent solubility index of up to 2.3-2.7 $\mathrm{mmol} / \mathrm{L}(138-162 \mathrm{mg} / \mathrm{L})$ at $37{ }^{\circ} \mathrm{C} .{ }^{16-18}$ If similar dissolution rates apply to the intact lung, it is possible that repetitive dosing could lead to biopersistence with a change in lung toxicity. While macrophage clearance could play a role in determining lung burden, it is important to consider that fumed silica is comprised of chain-like particles that are tightly embedded in the macrophage surface membrane without significant cellular uptake. The accompanying perturbation of ionic flux in the cell membrane has also been shown to induce potassium $\left(\mathrm{K}^{+}\right)$efflux, ${ }^{2}$ which acts as a trigger for assembly of the NLRP3 inflammasome and IL-1 $\beta$ production in macrophages. ${ }^{2,15,19}$ Membrane triggering differs from inflammasome activation by high aspect ratio materials (such as carbon nanotubes ${ }^{19}$ and metal oxide nanorods ${ }^{20}$ ) and a-quartz, ${ }^{15}$ which requires macrophage uptake and lysosomal damage as a prelude to the events leading to chronic lung damage. ${ }^{21,22}$ The shift in the mechanism of inflammasome activation (surface membrane $v s$. lysosomal damage) could play a role in fumed silica being able to induce sustained IL- $1 \beta$ release and sub-chronic lung damage, different from other forms of SAS. 
Against this background, we set out to investigate the impact of single-dose versus repetitive dose administration of fumed silica to compare the effects of NLRP3 activation in the lung and the possibility of sub-chronic lung damage. ${ }^{23} \mathrm{We}$ also endeavored to relate this research to the clearance and biopersistence of fumed silica nanoparticles, as well as the contribution of the reactive particle surfaces to sub-chronic injury. We demonstrate that only repetitive dosing could induce increased collagen deposition in the lung, compared to lack of an effect of a single-dose exposure, even at relatively high concentrations. Dosing frequency also impacted the dissolution and clearance rate of fumed silica. Surface silanol density was important in longer-term effects, as demonstrated by decreased lung fibrosis in response to titanium-doped fumed silica. We discuss the relevance of the data to the GRAS status of fumed silica.

\section{Results}

\section{Synthesis and Characterization of Fumed Silica Nanoparticles}

Flame spray pyrolysis (FSP) was used to synthesize pristine fumed silica nanoparticles as previously reported. ${ }^{2,24}$ The primary size of the chain-like particles was $16 \mathrm{~nm}$, as determined by TEM (Figure 1A). FTIR was used to assess the surface silanol density, which was previously identified as a key physicochemical parameter in the hazard potential of fumed silica nanoparticles. ${ }^{4}$ This yielded a silanol density of $12.86 \pm 1.87 \mathrm{OH} / \mathrm{nm}^{2}$ (Figure 1B). In addition to pristine particles, we also used FSP for the synthesis of Ti-doped fumed silica nanoparticles, which contained $2.4 \mathrm{wt} \% \mathrm{Ti}$ in the silica framework; doping reduced the silanol density to $7.63 \pm 2.38 \mathrm{OH} / \mathrm{nm}^{2}$ (Figure 1B). Dynamic light scattering (DLS) was used to assess the hydrodynamic sizes of the particles in water and a surrogate lung lining fluid (PBS supplemented with $0.6 \mathrm{mg} / \mathrm{mL}$ BSA and $0.01 \mathrm{mg} / \mathrm{mL}$ DPPC), which was used for pulmonary instillation. The dispersal of pristine and Ti-doped particles was better in PBS supplemented with BSA and DPPC than in water $(211 \mathrm{~nm}$ and $155 \mathrm{~nm} v s .221 \mathrm{~nm}$ and 252 $\mathrm{nm}$, respectively). This likely reflects the formation of a protein corona, which improves suspension stability (Figure 1B). ${ }^{25}$ Measurement of the zeta potential demonstrated negative charges for pristine and doped fumed silica in both exposure media $(\sim-30 \mathrm{mV}$ in water that was reduced to $\sim-10 \mathrm{mV}$ in surrogate lung lining fluid) (Figure 1B).

\section{Assessment of Sub-Chronic Pulmonary Hazard Potential and Particle Retention by Single vs. Repetitive Exposures}

We have recently demonstrated that fumed silica is capable of inducing acute lung inflammation due to the ability of the chain-like particles to induce cell membrane damage as well as the capability of the reactive silanol groups on the particle surface to catalyze ROS production. ${ }^{2}$ Interestingly, mechanistic studies also revealed that the particles are capable of tight binding to the cell surface membrane, where these particles are capable of inducing potassium efflux that serves as a stimulus for NLRP3 inflammasome activation. ${ }^{2,}{ }^{4}$ We have also previously demonstrated that high aspect ratio materials (e.g., carbon nanotubes ${ }^{19,26}$ ) and rare earth oxides ${ }^{26}$ are capable of activating the NLRP3 inflammasome, with the ability to induce sub-chronic pulmonary inflammation and lung fibrosis. While single-dose oropharyngeal instillation of fumed silica failed to induce noticeable fibrotic effects after 21 days in preliminary studies, we did observe evidence of an increased macrophage count in 
the bronchoalveolar (BAL) lavage fluid if the instilled dose was delivered as 3 separate doses, one week apart. In this communication, we used 3 bolus doses of 6,9 , and $21 \mathrm{mg} / \mathrm{kg}$ for comparison to the same amount of particles, delivered as 3 separate doses, with the same interval (i.e., $3 \times 2,3 \times 3$, and $3 \times 7 \mathrm{mg} / \mathrm{kg}$ ) (Figure $2 \mathrm{~A}$ ). The dosimetry calculations are based on an occupational exposure study in a silica manufacturing facility, where workers were exposed to $10.5 \mathrm{mg} / \mathrm{m}^{3}$ precipitated amorphous silica. ${ }^{23}$ This calculation is explained in Table S1 in the Supplement. Single-dose exposure was performed by oropharyngeal aspiration on day 0 , following which the animals were sacrificed on day 21 . Repetitive exposures were performed on days 0,7 and 14, with animal sacrifice on day 21 (Figure 2A). Aspiration of $5 \mathrm{mg} / \mathrm{kg}$ Min-U-Sil, a commercially available form of a-quartz (QTZ), was used as a positive control, while PBS supplemented with BSA and DPPC was used as a negative control.

Examination of the BAL fluid demonstrated that mice exposed to $3 \times 7 \mathrm{mg} / \mathrm{kg}$ pristine nanoparticles exhibit a significant increase of LIX (LPS-induced CXC chemokine), IL-1 $\beta$, and TGF- $\beta 1$ compared to mice receiving a one-time exposure of $21 \mathrm{mg} / \mathrm{kg}$ fumed silica (Figure 2B-D). Only QTZ induced an increase of similar amounts of LIX and TGF- $\beta 1$ as the animals exposed to $3 \times 7 \mathrm{mg} / \mathrm{kg}$ fumed silica. TGF- $\beta 1$ is a biomarker for sub-chronic lung injury in the setting of epithelial-mesenchymal transition (EMT), leading to pro-fibrogenic effects. $^{27,28}$ One source of TGF- $\beta 1$ is likely the epithelial cells, as demonstrated in our previous studies. ${ }^{29,30}$ Indeed, assessment of the whole lung collagen content by the Sircol assay confirmed increased collagen deposition in response to a single dose of QTZ or triplicate dosing with 3 and $7 \mathrm{mg} / \mathrm{kg}$ fumed silica (Figure 2E). This was confirmed by Trichrome staining, which showed peribronchial collagen deposition in animals treated with QTZ, as well as exposure to $3 \times 3$ or $3 \times 7 \mathrm{mg} / \mathrm{kg}$ fumed silica (Figure $2 \mathrm{~F}$ ).

Lung tissue was used to measure the Si content by ICP-OES. This demonstrated that onetime exposure to $21 \mathrm{mg} / \mathrm{kg}$ fumed silica is largely cleared by day 21 , with the retention of only $0.22 \mathrm{mg} \mathrm{Si} / \mathrm{mg}$ of lung extract protein (Figure $2 \mathrm{G}$ ). In contrast, the comparable values were 0.65 and $4.12 \mathrm{mg} \mathrm{Si} / \mathrm{mg}$ protein for instillation of $3 \times 7 \mathrm{mg} / \mathrm{kg}$ fumed silica or $1 \times 5$ $\mathrm{mg} / \mathrm{kg}$ QTZ, respectively (Figure $2 \mathrm{G}$ ). All other fumed silica challenges failed to achieve significant lung retention. While removal of fumed silica and quartz by the mucociliary escalator $^{31}$ and macrophage uptake ${ }^{30}$ could contribute to the differences in lung retention, a more likely explanation are differences in particle dissolution. ${ }^{32}$ Since SAS materials are known to dissolve to various degrees in aquatic solution, $16,17,33$ the dissolution of fumed silica was assessed in water, simulated lung interstitial fluid (Gamble's solution), surrogate lung lining fluid (SLF4), oropharyngeal instillation fluid (PBS supplemented with 0.6 $\mathrm{mg} / \mathrm{mL}$ BSA and $0.01 \mathrm{mg} / \mathrm{mL}$ DPPC), and RPMI 1640 cell culture medium, supplemented with $10 \%$ FBS (Figure 3A). Following particle incubation in these fluids for 0,6 and $40 \mathrm{~h}$ and the collection of supernatants and pellets, ICP-OES analysis demonstrated that while fumed silica nanoparticles were more stable in water, $>25 \%$ of the particle mass dissolved within $6 \mathrm{~h}$ in Gamble's solution, surrogate lung lining fluid, oropharyngeal instillation fluid and cell culture medium (Figure 3B). Dissolution slowed after $6 \mathrm{~h}$, most likely as a result of saturation in the system (Figure 3B). TEM analysis demonstrated progressive fusing of the nanoparticles with diminished-size over time, resulting in the formation of large agglomerates in Gamble's solution (Figure S1). These results support the notion that the 
dissolution of fumed silica plays an important role in lung clearance. In order to assess the role of particle dissolution in the activation of the NLRP3 inflammasome, particle remnants and supernatants were collected in Gamble's solution (after $40 \mathrm{~h}$ of suspension) for analysis in THP-1 cells (Figure S2). While the supernatants, presumably soluble silicic acid and polysilicic acid dissolution by-products proven to be non-toxic, ${ }^{34}$ did not stimulate IL-1 $\beta$ production, the particle remnants were associated with increased cytokine production (Figure S2). This effect was blunted when particle remnants from Ti-doped fumed silica nanoparticles were used, indicating the important role of high silanol density on the surface of the particle remnants (Figure S2). In summary, the above data indicate that, even if dosing levels remain the same, repetitive exposure to fumed silica leads to a change in fumed silica particle retention and pro-inflammatory effects in the lung. What are the molecular and cellular explanations for these in vivo differences?

\section{Comparative Analysis of the Cellular and Cytokine Response Kinetics of Single vs. Repetitive Fumed Silica Exposures}

A kinetics study was designed to compare exposure to $3 \times 7 \mathrm{mg} / \mathrm{kg}$ (days 0,7 and 14) fumed silica with a single $21 \mathrm{mg} / \mathrm{kg}$ dose, instilled on day 0 (Figure $4 \mathrm{~A}$ ). The animals were sacrificed $40 \mathrm{~h}$ following each of the instillations as well as on day 21. Differential cell counts showed continuous macrophage recruitment to the BAL fluid during repetitive dosing of fumed silica (Figure 4B). This resulted in a progressive increase in the total macrophage count over the 21 day observation period. In contrast, macrophage recruitment peaked on day 7 and then gradually tapered-off over the ensuing 14 days after administration of a single dose of fumed silica (Figure 4B). QTZ induced a stable elevation of the macrophage cell count from days 7-21 (Figure 4B). Repetitive dosing also induced elevated levels of LIX from days 7-21 (Figure 4C). Single dose exposure had a negligible effect on this cytokine, while QTZ induced a significant and sustained increase over 21 days (Figure 4C). While IL- $1 \beta$ levels were increased for all the exposures upon the first instillation, there was a clear difference between single and repetitive dosing over the ensuing observation period (Figure 4D). Thus, while there was a progressive decline in the IL-1 $\beta$ levels over 21 days after single dose exposure, there was a progressive rise in cytokine levels upon repetitive dosing. IL- $1 \beta$ levels were consistently elevated following QTZ instillation. A similar trend and differences between single $v s$. repetitive dose fumed silica exposure were seen during assaying of the pro-fibrogenic growth factor, TGF- $\beta 1$ (Figure 4E). Similar data for macrophage inflammatory protein-1a (MIP-1a), IL-6 and macrophage-derived chemokine (MDC) are shown in the supplementary data section (Figures S3 A-C). Overall these results demonstrate a tendency for pro-inflammatory markers to remain elevated during repetitive but not single dose exposures.

Histological analysis (H\&E staining) showed that repetitive administration of fumed silica and single-dose exposures to QTZ are capable of inducing sustained focal inflammation in the lung (Figure S4). In contrast, a single high dose of fumed silica induced peribronchial inflammation at $40 \mathrm{~h}$ and 7 days that disappeared from day 14 to day 21. Repetitive fumed silica challenges also induced prominent collagen deposition around the small airways after 21 days, as shown by Masson's Trichrome staining (Figure 5A). QTZ had the same effect. In contrast, single dose exposure to fumed silica did not lead to collagen deposition (Figure 
5A). The Trichrome staining results were confirmed by a quantitative Sircol collagen assay (Figure 5B).

Lung tissue was used for determining Si distribution and content. The first approach was to label the fumed silica particles with the near-infrared (NIR) dye, DyLight680, followed by IVIS imaging of the explanted organs, including the lung (Figure 6A). Viewing of the NIR fluorescence intensities over time demonstrated that the fumed silica nanoparticles remain primarily confined to the lung. While a prominent signal could be detected in the lung $40 \mathrm{~h}$ after a single dose of $21 \mathrm{mg} / \mathrm{kg}$, the labeled particles were completely cleared after 7 days. In contrast, repetitive exposure to $3 \times 7 \mathrm{mg} / \mathrm{kg}$ fumed silica could be seen to prolong the signal intensity for up to 14 days (Figure 6A). Calculation of the fluorescence intensity in the region of interest was consistent with ICP-OES analysis of Si content in the lung (Figures 6B and 6C). Additional immunohistochemical analysis and fluorescence microscopy was performed to visualize the cellular distribution of the labeled particles in a relationship to FITC-labeled macrophages in the lung (Figure 6D). This demonstrated significant macrophage recruitment (green) to the interstitial lung tissue $40 \mathrm{~h}$ after instillation of $1 \times 21$ $\mathrm{mg} / \mathrm{kg}$ or $1 \times 7 \mathrm{mg} / \mathrm{kg}$ labeled fumed silica (Figure $6 \mathrm{D}$ ). The cellular recruitment was accompanied by the presence of NIR-labeled particles in the same tissue compartments (Figure 6A). However, while there was a rapid decline in macrophage recruitment to the lungs of mice receiving a one-time exposure, there was prominent macrophage retention for the entire observation period of 21 days in animals during repetitive dosing (Figure 6D).

\section{Titanium Doping Ameliorates Sub-Chronic Pulmonary Fibrosis}

We have previously demonstrated that Ti-doping decreases the acute hazard potential of fumed silica by decreasing the density of surface reactive silanol groups and the potential to generate ROS. ${ }^{2}$ Since this safer design approach also reduces acute pulmonary toxicity, ${ }^{2}$ we asked whether doping would affect the sub-chronic pro-fibrogenic potential of fumed silica in the lung. While Ti doping does not significantly affect the rate of fumed silica dissolution (Figure S1A and B), it decreases the surface silanol density (Figure 1B) as well as IL-1 $\beta$ production in THP-1 cells (Figure S2). When tested in the murine lung, repetitive exposure to $3 \times 7 \mathrm{mg} / \mathrm{kg}$ non-doped or doped fumed silica, it could be seen that doping significantly suppress IL-1 $\beta$ (Figure 7A), LIX (Figure 7B), and TGF- $\beta 1$ production (Figure 7C) relative to pristine fumed silica and quartz. As demonstrated in Figure 7A-C, a $1 \times 21 \mathrm{mg} / \mathrm{kg}$ dose did not induce cytokine and growth factor production. The protective effect of doping was also confirmed by reduced collagen deposition in the lung, using the Sircol assay (Figure 7D) as well as Masson's Trichrome staining (Figure 7E). Doping also reduced focal inflammation of fumed silica particles as determined by H\&E staining (Figure S5). In summary, Ti-doping appears to be equally effective for reducing the acute and chronic pulmonary effects of fumed silica. This suggests that the change in particle clearance with repetitive dosing remains dependent on surface silanol expression in triggering sub-chronic lung damage.

\section{Discussion}

In this communication, we demonstrate that fumed silica exhibits sub-chronic injury potential in the lung during repetitive dosing. Repetitive dosing is responsible for persistent 
recruitment of macrophages and the activation of the NLRP3 inflammasome. Single dose administration does not pose the same hazard, likely due to the comparatively high rate of dissolution and clearance of fumed silica from the lung. In contrast, repetitive dosing leads to biopersistence of the chain-like fumed silica particles, which have the capacity to trigger the assembly of the NLRP3 inflammasome subunits by a mechanism that differs from high aspect ratio materials and crystalline silica. The unique physicochemical properties of fumed silica vis-à-vis other SAS, including its chain-like morphology and high density display of reactive silanols, and ability to generate ROS, appear to be equally important for the subchronic as for the acute hazard potential in the lung. This is confirmed by the reduction in collagen deposition in the lung if titanium-doped fumed silica particles are used, which both reduce the $\mathrm{OH}$ density and the potential to generate ROS. ${ }^{2}$ Thus, fumed silica exhibits structure-activity relationships that require re-consideration of its GRAS status during chronic inhalation exposure. As a result of its increased biopersistence during repetitive exposure, the ability of even the remnant fumed silica particles to continuously activate the NLRP3 inflammasome is capable of inducing pro-fibrogenic effects in the lung.

The major finding of this study is that repetitive fumed silica exposure is capable of switching the inflammatory response in the lung from a transient, acute and reversible response mode to sub-chronic and persistent inflammation with definitive pro-fibrogenic potential. In addition to the role of the reactive surface of fumed silica in being able to generate acute membrane damage and oxidative stress (a key driver of acute inflammation), ${ }^{2,4}$ additional structure-activity relationships come into play during the subchronic stage. These include the unique impact of the fumed silica composition in terms of biopersistence, activation of the NLRP 3 inflammasome and IL-1 $\beta$ production. First, in addition to the highly reactive and $\mathrm{H}$-bonded surface silanols that are generated during opening of the highly energetic three-membered siloxane rings (resulting from high temperature synthesis), ${ }^{4}$ the chain-like structure of the particles as well as the kinetics of dissolution need to be considered during repetitive exposure conditions. The fractal chainlike structure of fumed silica (unique among SAS materials) allows tight adherence to cell surface membranes in the lung, with the possibility of interfering in uptake by macrophages. Additionally, due to its high surface area, highly reactive surface silanol, and strained threemembered rings, fumed silica NPs generate relatively high concentrations of surfaceassociated radicals that are capable of ROS generation compared to other forms of SAS such as Stober silica. ${ }^{4}$ This unique membrane interaction and ROS generation also leads to cellular lysis, as we have demonstrated in hemolysis assays, ${ }^{4}$ as well as perturbation of ionic flux in the macrophage membrane. ${ }^{2}$ This could induce $\mathrm{K}^{+}$efflux, which in the company of oxygen radical generation in the membrane, triggers assembly of NLRP3 inflammasome components. $^{2}$ This scenario leads to a unique mode of activation of the inflammasome compared to other nanomaterials that can engage this adverse outcome pathway, including a-quartz ${ }^{15}$, high aspect ratio materials such as carbon nanotubes ${ }^{19}$ or rare earth oxide nanoparticles. ${ }^{26}$ The latter group of materials all require cellular uptake and subcellular processing by lysosomes, which can be damaged by particle characteristics such as shape ${ }^{35,36}$ and surface catalytic activity. ${ }^{19}$ The release of hydrolytic enzymes (such as cathepsin B) and oxidative stress plays a role in intracellular assembly of the NLRP3 inflammasome in the vicinity of the damaged lysosomes. ${ }^{37,38}$ While we have shown 
previously that the alternative membrane-initiated pathway for inflammasome activation is instrumental in IL- $1 \beta$ production by fumed silica, ${ }^{2}$ our original observation suggested that this is a transient event only. However, the data emerging from this study is that the inflammasome activation can be sustained by repetitive dosing, thereby introducing another variable that needs to be considered when evaluating the GRAS status of fumed silica.

The increased biopersistence as a result of repetitive dosing is intimately linked to the alteration of particle clearance characteristics. It has been reported that different forms of silica, including quartz, amorphous, and mesoporous silica, exhibit different rates of hydrolysis during aquatic suspension, leading to formation of silicic acid. ${ }^{39}$ In addition to material composition, the dissolution of silica nanoparticles is dependent on particle size, $\mathrm{pH}$, state of internal hydration and the presence of trace amounts of impurities in the silica matrix or absorbed on its surface. ${ }^{40}$ Our data confirm that quartz exhibit little dissolution while up to $30 \%$ of fumed silica undergo dissolution during 6 hours suspension in simulated biological fluids (Figure 3B\&S1). We have also previously demonstrated that fumed silica has a different rate of dissolution from Stöber silica in simulated lung lining fluid (data not shown). The current study explores the linkage between biopersistence and fumed silica dosing frequency. While particle uptake by macrophages may also contribute to clearance, ${ }^{41}$ it is interesting that the tight binding of fumed silica to the macrophage surface membrane has a restraining effect on particle phagocytosis. ${ }^{2,4}$ This is consistent with reports showing few or no particle-containing macrophages in the lungs of the guinea pigs and rats after fumed silica exposure. ${ }^{13}$ While it is unknown to what extent the surface membrane interactions impact the rate of macrophage clearance, we know that surface silanol density is important in sustaining the pro-fibrogenic pathway, as demonstrated by the impact of titanium doping. In contrast, the spherical shape and lesser surface reactivity of other forms of SAS (e.g., Stöber silica) do not interfere in macrophage uptake, which increases the possibility of uptake into the acidic phagolysosomal environment ( $\mathrm{pH} 4.5),{ }^{42,} 43$ where amorphous silica has appreciable solubility. ${ }^{40}$ However, while a-quartz is also taken up into the phagolysosomal compartment, the much lower rate of dissolution in an acidic environment does not substantially impact biopersistence. This explains why in the current study a single dose of QTZ was sufficient for inducing persistent activation of the NLRP3 pathway and pulmonary fibrosis.

The fact that fumed silica could induce chronic lung toxicity after repetitive dosing should raise awareness of this material's GRAS status. This is an important consideration based on the high production volumes of fumed silica and its widespread use in food, pharmaceuticals, cosmetics, tires, printer toners, coatings, paints, anti-caking agents, etc. ${ }^{1}$ In addition to inhalation exposure, it is also important to ponder the effects of gastrointestinal tract (GIT), skin, and eye contact. This should include consideration as to whether the potential hazardous impact on each of these target organs is equivalent. Also, fumed silica intake by the GIT could lead to particle uptake and dissemination to the liver, kidney and spleen. ${ }^{44}$ It has been demonstrated, for instance, that long term oral intake of fumed silica could lead to liver fibrosis in rats. ${ }^{44}$ It will be particularly interesting to see whether the use of titanium-doped nanoparticles can ameliorate this effect, as demonstrated in the lung. Since a large number of SAS studies failed to identify the specific type of silica (e.g., Stöber, fumed or precipitated silica) ${ }^{11}$ or have not comprehensively investigated physicochemical 
properties such as siloxane ring structure, hydration status, surface silanol density, single spherical versus chain-like particles, in vitro and in vivo dissolution rates, etc, we opine that further studies are necessary to explore the impact of these parameters on fumed silica safety. This should also address whether the unique characteristics of this material should receive scrutiny towards regulatory decision-making. It is particularly important to consider performance of aerosolized inhalation to demonstrate whether our mechanistic studies are relevant under more realistic exposure conditions.

\section{Conclusion}

In this study, we demonstrate that repetitive fumed silica dosing induced sub-chronic lung injury while single bolus dose exposure did not show the same effect. The difference is likely due to the change in fumed silica clearance, leading to biopersistence and continuous triggering of inflammasome activation. These adverse effects could be reduced by titanium doping of fumed silica, suggesting its potential as a safer-by-design alternative. Our data raise consciousness of whether fumed silica can be GRAS or whether additional investigation is required to determine its long-term hazard potential during chronic exposure.

\section{EXPERIMENTAL SECTION}

\section{Reagents and Materials}

Crystalline silica, MIN-U-SIL (MUS, $5 \mu \mathrm{m}$ in size), was kindly provided by US SILICA (Berkeley Springs, WV). The ELISA kits for human IL-1 $\beta$ and mouse LIX were purchased from R\&D Systems (Minneapolis, MN); the ELISA kits for mouse IL-1 $\beta$, MIP-1a, IL-6, MDC and TGF- $\beta 1$ were purchased from BD Biosciences (San Diego, CA).

\section{Synthesis of Pristine and Doped Fumed Silica Nanoparticles}

The pristine and titanium (Ti)-doped fumed silica nanoparticles were synthesized using flame spray pyrolysis (FSP). ${ }^{2}, 45-47$ The required amounts of tetraethylorthosilicate (TEOS, Si-precursor, Sigma Aldrich) and titanium isopropoxide (Ti-precursor, Sigma Aldrich) were mixed together with xylene to provide $7 \%$ Ti by weight before combustion. ${ }^{2}$ During flame spray pyrolysis, the liquid precursors were delivered at a rate of $5 \mathrm{~mL} / \mathrm{min}$ using a syringe pump and were atomized using $5 \mathrm{~L} / \mathrm{min}_{2}$ at a constant pressure drop of $1.5 \mathrm{bar}$ at the nozzle tip. ${ }^{2}$ The spray was ignited by a premixed co-delivery of $\mathrm{CH}_{4}$ and $\mathrm{O}_{2}(1.5 \mathrm{~L} / \mathrm{min}, 3.2$ $\mathrm{L} / \mathrm{min}$ ) forming a spray flame (Figure 1A). The ultrafine particles were formed by reaction, nucleation, surface growth, coagulation and coalescence in the flame environment. ${ }^{48}$ The particles were collected from a $257 \mathrm{~mm}$ glass filter placed in the flame reactor at the distance of $60 \mathrm{~cm}$ from the flame nozzle.

\section{Characterization of Fumed Silica}

Fourier transformed infrared (FTIR) spectra were recorded by a Bruker Vertex-70 FTIR spectrometer, using the $\mathrm{KBr}$ pellet technique to determine total silanol display. ${ }^{4} \mathrm{High}$ throughput dynamic light scattering (HT-DLS, Dynapro ${ }^{\text {TM }}$ Plate Reader, Wyatt Technology) was performed to determine the particle size and size distribution of the fumed silica nanoparticles in water and PBS supplemented with BSA and DPPC following our well- 
established protocol. ${ }^{49}$ Zeta potential measurement was conducted in a Brookhaven ZetaPALS instrument.

\section{NIR labeling}

The NIR fluorescent dye, DyLight 680 NHS ester, was used for particle labeling. ${ }^{50}$ Briefly, ten milligram of particles were suspended in $1 \mathrm{~mL}$ of ethanol and mixed with $10 \mu \mathrm{L}$ of (3aminopropyl)triethoxysilane. The reaction took place under an inert atmosphere with consistent stirring at $80^{\circ} \mathrm{C} .12 \mathrm{~h}$ later, the mixture was centrifuged and washed with ethanol for several times. Then, the particles were suspended in $1 \mathrm{~mL}$ of DMF and mixed with 0.1 mg of DyLight 680 NHS ester with stirring at room temperature for $2 \mathrm{~h}$. The particles were centrifuged and washed with ethanol and deionized water before use.

\section{Dispersion of Fumed Silica in Exposure Media}

Fumed silica was provided as dry powders. The samples were weighed in a fume hood on an analytical balance and suspended in deionized water at a concentration of $5 \mathrm{mg} / \mathrm{mL}$. These suspensions were sonicated for $15 \mathrm{~min}$ in a water sonicator bath (Branson, Danbury, CT, USA, model 2510, $100 \mathrm{~W}$ output power; $42 \mathrm{kHz}$ frequency). ${ }^{49}$ An appropriate amount of fumed silica from each stock solution was added to the desired final concentration in exposure media including cell culture media or PBS supplemented with BSA $(0.6 \mathrm{mg} / \mathrm{mL})$ and DPPC $(0.01 \mathrm{mg} / \mathrm{mL}) .{ }^{51}$ The diluted particle suspensions were sonicated for $15 \mathrm{~min}$ to obtain well-dispersed particle suspensions at the desired final concentrations.

\section{Exposure of Fumed Silica Nanoparticles by Oropharyngeal Aspiration and Assessment of Exposure Outcomes}

Eight week old male C57BL/6 mice were purchased from Charles River Laboratories (Hollister, CA). All animals were housed under standard laboratory conditions that have been set up according to UCLA guidelines for care and treatment of laboratory animals as well as the NIH guide for the care and use of laboratory animals in research (DHEW78-23). ${ }^{51}$ These conditions are approved by the Chancellor's Animal Research Committee at UCLA and include standard operating procedures for animal housing (filtertopped cages; room temperature at $23 \pm 2{ }^{\circ} \mathrm{C} ; 60 \%$ relative humidity; $12 \mathrm{~h}$ light, $12 \mathrm{~h}$ dark cycle) and hygiene status (autoclaved food and acidified water). Animal exposure to fumed silica was carried out by an oropharyngeal aspiration method as described at NIOSH. ${ }^{52}$ Briefly, animals were anesthetized by intraperitoneal (i.p.) injection of ketamine (100 $\mathrm{mg} / \mathrm{kg}) / x y l a z i n e(10 \mathrm{mg} / \mathrm{kg})$ in a total volume of $100 \mu \mathrm{L}$. With the anesthetized animals held in a vertical position, $50 \mu \mathrm{L}$ of a PBS suspension (containing certain the indicated quantity of pristine or doped fumed silica) was instilled at the back of the tongue for pulmonary aspiration. ${ }^{52}$ For the repetitive dosing study, C57BL/6 mice were exposed to either 3 doses of 2,3 , and $7 \mathrm{mg} / \mathrm{kg}$ one week apart (i.e., $3 \times 2,3 \times 3$, and $3 \times 7 \mathrm{mg} / \mathrm{kg}$ ), compared to singledose deliveries of 6,9 , and $21 \mathrm{mg} / \mathrm{kg}$ by oropharyngeal aspiration (Figure $2 \mathrm{~A}$ ). The animals were sacrificed on day 21. For the kinetic study, the exposure effects of $3 \times 7 \mathrm{mg} / \mathrm{kg}$ (on days 0,7 and 14) fumed silica was compared to $1 \times 21 \mathrm{mg} / \mathrm{kg}$ instillation on day 0 . The animals were sacrificed $40 \mathrm{~h}$ after each day of instillation as well as on day 21 (Figure 4A). Bronchoalveolar lavage (BAL) fluid and lung tissues were collected as previously described. ${ }^{53}$ Briefly, the trachea was cannulated and the lungs were gently lavaged three 
times with $1 \mathrm{~mL}$ of sterile PBS to obtain BAL fluid. The BAL fluid was used for total and differential cell counting and to measure IL-1 $\beta$, TGF- $\beta 1$, MIP-1 $\alpha$, MDC, IL- 6 and LIX levels. Lung tissues were collected and stained with hematoxylin/eosin or with Masson's Trichrome staining. IL-1 $\beta$, TGF- $\beta 1$, MIP-1 $\alpha$, MDC, IL- 6 were measured by the ELISA kits.

\section{Immunohistochemistry}

Mouse lungs were examined by immunohistochemistry (IHC) to determine the distribution of pulmonary macrophages and NIR-labeled fumed silica nanoparticles. Lung tissue was fixed with ice-cold acetone, acetone/chloroform $(1: 1, \mathrm{v} / \mathrm{v})$, and acetone for 5 minutes at room temperature, respectively. The sample was incubated with primary anti-F4/80 antibody $\left(1 / 200\right.$ in PBS) at $4{ }^{\circ} \mathrm{C}$ for 16 hours. Alexa Fluor ${ }^{\circledR} 488$ goat anti-rat (1/200 in PBS) was used as the secondary antibody. Slides were visualized under a Leica confocal SP2 1P-FCS microscope (Advanced Light Microscopy/Spectroscopy Shared Facility, UCLA). Highmagnification images were obtained with a $63 \times$ objective (Leica, N.A.=1.4).

\section{Fumed Silica Biodistribution}

IVIS optical imaging (Xenogen) was used to study the biodistribution of NIR-labeled fumed silica in exposed mice. The exposure effect of $3 \times 7 \mathrm{mg} / \mathrm{kg}$ (on days 0,7 and 14) fumed silica was compared to a $1 \times 21 \mathrm{mg} / \mathrm{kg}$ instillation on day 0 . The animals were sacrificed $40 \mathrm{~h}$ after each day of instillation as well as on day 21 (Figure 4A). Fluorescence images were captured after animals were sacrificed and major organs were collected. Lung tissues were also collected for the measurement of the Si content using ICP-OES.

\section{Fumed Silica Dissolution in Exposure Media}

Inductively coupled plasma optical emission spectrometry (ICP-OES) analysis was used to detect the Si content of particles suspended in different solutions (Figure 3A). The suspension of pristine and 7\% Ti-doped fumed silica nanoparticles were prepared in $1 \mathrm{~mL}$ at $100 \mu \mathrm{g} / \mathrm{mL}$ in deionized water, simulated lung fluids, including Gamble's solution and SLF4, surrogate lung lining fluid (PBS supplemented with $0.6 \mathrm{mg} / \mathrm{mL}$ BSA and $0.01 \mathrm{mg} / \mathrm{mL}$ DPPC), and RPMI 1640 plus $10 \%$ FBS. All suspensions were incubated at $37{ }^{\circ} \mathrm{C}$ in a $5 \%$ $\mathrm{CO}_{2}$ atmosphere and each $1 \mathrm{~mL}$ of suspension were centrifuged at $15,000 \mathrm{~g}$ for $60 \mathrm{~min}$ at different time points $(0,6$ and $40 \mathrm{hr})$. The supernatants of each sample were transferred to clean tubes (SC475, Environmental Express) for acid digestion. Digestion was carried out with $10 \mathrm{~mL}$ of concentrated $\mathrm{HNO}_{3}\left(65-70 \%\right.$, Trace Metal Grade, Fisher Scientific) at $95{ }^{\circ} \mathrm{C}$ overnight in a HotBlock (SC100, Environmental Express). ${ }^{54,55}$ Once all digested solutions were dried out, the samples were cooled to room temperature and subsequently diluted by $2 \%(\mathrm{v} / \mathrm{v})$ nitric acid at $95{ }^{\circ} \mathrm{C}$ for $3 \mathrm{~h}$ to extract the analytes. These extracts were transferred to $15 \mathrm{~mL}$ ICP-OES analysis tubes and additional $2 \%$ nitric acid was added to reach a final volume of $8 \mathrm{~mL}$. Each exposure medium in the absence of fumed silica nanoparticles was also digested as a blank reagent. The Si content was quantified using an ICP-OES (ICPE-9000, Shimadzu, Japan) and a calibration curve was established using a standard $\mathrm{Si}$ solution (AccuStandard, $1,000 \mathrm{mg} / \mathrm{L}$ in $2 \% \mathrm{HNO}_{3}$ ). Each sample and standard was analyzed in triplicate in the presence of $2 \%(\mathrm{v} / \mathrm{v})$ nitric acid. 


\section{Sircol Collagen Assay}

Collagen contents in murine lungs were quantified using a Sircol collagen assay according to manufacturer's protocol. Briefly, lung tissues were broken down in PBS buffer using a probe sonicator at $0{ }^{\circ} \mathrm{C}^{2,51}$ Then the tissue debris was treated in $500 \mu \mathrm{L}$ of acetic acid $(0.5$ M) with pepsin $(0.1 \mathrm{mg} / \mathrm{mL})$ at $4{ }^{\circ} \mathrm{C}$ for overnight. Following centrifugation at $3000 \mathrm{rpm}$ for $10 \mathrm{~min}$, the supernatants were collected to measure the total protein concentration. The supernatant was then treated with $50 \mu \mathrm{L}$ of acid neutralising reagent plus $100 \mu \mathrm{L}$ of collagen isolation \& concentration reagent at $4{ }^{\circ} \mathrm{C}$ for overnight. On the third day, the reactant was treated with $0.5 \mathrm{~mL}$ of Sircol Dye Reagent and was placed on a shaker by inverting the content. 30 min later, the reactant was centrifuged at 12,000 rpm for $10 \mathrm{~min}$ and the pellet was harvested. Then, $375 \mu \mathrm{L}$ of ice-cold acid-salt wash reagent was added to the pellet to remove unbound dye. Following another centrifugation at $12000 \mathrm{rpm}$ for $10 \mathrm{~min}$, the supernatant was drained and the remaining dye was blotted. Further, $125 \mu \mathrm{L}$ of alkali reagent was used to dissolve the pellet within $5 \mathrm{~min}$. The absorption at $555 \mathrm{~nm}$ was determined using a SpectraMax M5 microplate reader (Molecular Devices, Sunnyvale, CA). The collagen concentration of samples was determined using the standard curve constructed with known concentrations of collagen.

\section{Statistical Analysis}

All the experiments were repeated two to three times. For all the figures and tables, the values shown represent mean \pm SD. Statistical significance was determined by two-tailed Student's t-test for two-group analysis.

\section{Supplementary Material}

Refer to Web version on PubMed Central for supplementary material.

\section{Acknowledgments}

This work was primarily supported by the US Public Health Service Grant, R01 ES016746, and leveraged support from the National Science Foundation and the Environmental Protection Agency under Cooperative Agreement Number DBI 0830117 and 1266377. Any opinions, findings, and conclusions or recommendations expressed in this material are those of the author(s) and do not necessarily reflect the views of the National Science Foundation or the Environmental Protection Agency. This work has not been subjected to EPA review and no official endorsement should be inferred. The authors would thank the CNSI Advanced Light Microscopy/Spectroscopy Shared Facility at UCLA for confocal fluorescent microscopy, the use of TEM instruments at the Electron Imaging Center for NanoMachines supported by NIH (1S10RR23057 to Z.H.Z.) and CNSI at UCLA

\section{References}

1. Trommer RM, Bergmann CP. Flame Spray Technology: Method for Production of Nanopowders. 2015:80.

2. Sun B, Pokhrel S, Dunphy DR, Zhang H, Ji Z, Wang X, Wang M, Liao Y-P, Chang CH, Dong J, Li R, Mädler L, Brinker CJ, Nel AE, Xia T. Reduction of Acute Inflammatory Effects of Fumed Silica Nanoparticles in the Lung by Adjusting Silanol Display through Calcination and Metal Doping. ACS Nano. 2015; 9:9357-9372. [PubMed: 26200133]

3. Maynard AD. Old Materials, New Challenges? Nat. Nanotechnol. 2014; 9:658-659. [PubMed: 25182032]

4. Zhang H, Dunphy D, Jiang X, Meng H, Sun B, Tarn D, Xue M, Wang X, Lin S, Ji Z, Li R, Garcia F, Yang J, Kirk M, Xia T, Zink J, Nel A, Brinker C. Processing Pathway Dependence of Amorphous 
Silica Nanoparticle Toxicity: Colloidal vs Pyrolytic. J. Am. Chem. Soc. 2012; 134:15790-15804. [PubMed: 22924492]

5. Nash T, Allison AC, Harington JS. Physico-Chemical Properties of Silica in Relation to its Toxicity. Nature. 1966; 210:259-261. [PubMed: 4289018]

6. Marks J. The Neutralization of Silica Toxicity In Vitro. Br. J. Ind. Med. 1957; 14:81-84. [PubMed: 13426429]

7. Vitums VC, Edwards MJ, Niles NR, Borman JO, Lowry RD. Pulmonary Fibrosis from Amorphous Silica Dust, A Product of Silica Vapor. Arch. Environ. Health. 1977; 32:62-68. [PubMed: 192157]

8. Harber P, Dahlgren J, Bunn W, Lockey J, Chase G. Radiographic and Spirometric Findings in Diatomaceous Earth Workers. J. Occup. Environ. Med. 1998; 40:22-28. [PubMed: 9467117]

9. Groth, DH.; Moorman, WJ.; Lynch, DW.; Stettler, LE.; Wagner, WD.; Hornung, RW. Chronic Effects of Inhaled Amorphous Silicas in Animals. Health Effects of Synthetic Silica Particulates, ASTM Special Technical Publication 732 ed. Philadelphia, PA: American Society for Testing and Materials; 1981.

10. Chen Z, Meng H, Xing G, Yuan H, Zhao F, Liu R, Chang X, Gao X, Wang T, Jia G, Ye C, Chai Z, Zhao Y. Age-Related Differences in Pulmonary and Cardiovascular Responses to SiO2 Nanoparticle Inhalation: Nanotoxicity Has Susceptible Population. Environ. Sci. Technol. 2008; 42:8985-8992. [PubMed: 19192829]

11. Napierska D, Thomassen L, Lison D, Martens J, Hoet P. The Nanosilica Hazard: Another Variable Entity. Part. Fibre Toxicol. 2010; 7:39. [PubMed: 21126379]

12. Schepers GW, Delahant AB, Schmidt JG, Vonwecheln JC, Creedon FT, Clark RW. The Biological Action of Degussa Submicron Amorphous Silica Dust (Dow Corning Silica). III. Inhalation Studies on Rabbits. AMA Arch. Ind. Health. 1957; 16:280-301. [PubMed: 13457449]

13. Warheit DB. Inhaled Amorphous Silica Particulates: What Do We Know About Their Toxicological Profiles? J. Environ. Pathol., Toxicol. Oncol. 2001; 1:133-141.

14. Schepers GW, Durkan TM, Delahant AB, Creedon FT, Redlin AJ. The Biological Action of Degussa Submicron Amorphous Silica Dust (Dow Corning Silica). I. Inhalation Studies on Rats. AMA Arch. Ind. Health. 1957; 16:125-146. [PubMed: 13443529]

15. Dostert C, Petrilli V, Van Bruggen R, Steele C, Mossman BT, Tschopp J. Innate Immune Activation through Nalp3 Inflammasome Sensing of Asbestos and Silica. Science. 2008; 320:674677. [PubMed: 18403674]

16. Roelofs F, Vogelsberger W. Dissolution Kinetics of Synthetic Amorphous Silica in Biological-Like Media and Its Theoretical Description. J. Phys. Chem. B. 2004; 108:11308-11316.

17. He Q, Shi J, Zhu M, Chen Y, Chen F. The Three-Stage In Vitro Degradation Behavior of Mesoporous Silica in Simulated Body Fluid. Microporous Mesoporous Mater. 2010; 131:314-320.

18. Gun'ko VM, Mironyuk IF, Zarko VI, Voronin EF, Turov VV, Pakhlov EM, Goncharuk EV, Nychiporuk YM, Vlasova NN, Gorbik PP, Mishchuk OA, Chuiko AA, Kulik TV, Palyanytsya BB, Pakhovchishin SV, Skubiszewska-Zieba J, Janusz W, Turov AV, Leboda R. Morphology and Surface Properties of Fumed Silicas. J. Colloid Interface Sci. 2005; 289:427-445. [PubMed: 16024031]

19. Wang X, Xia T, Duch M, Ji Z, Zhang H, Li R, Sun B, Lin S, Meng H, Liao Y-P, Wang M, Song TB, Yang Y, Hersam M, Nel A. Pluronic F108 Coating Decreases the Lung Fibrosis Potential of Multiwall Carbon Nanotubes by Reducing Lysosomal Injury. Nano Lett. 2012; 12:3050-3061. [PubMed: 22546002]

20. Lin S, Wang X, Ji Z, Chang CH, Dong Y, Meng H, Liao Y-P, Wang M, Song T-B, Kohan S, Xia T, Zink JI, Lin S, Nel AE. Aspect Ratio Plays a Role in the Hazard Potential of CeO2 Nanoparticles in Mouse Lung and Zebrafish Gastrointestinal Tract. ACS Nano. 2014; 8:4450-4464. [PubMed: 24720650]

21. Borm P, Cassee F, Oberdorster G. Lung Particle Overload: Old School -New Insights? Part. Fibre Toxicol. 2015; 12:10. [PubMed: 25927223]

22. Bonner JC. Lung Fibrotic Responses to Particle Exposure. Toxicol. Pathol. 2007; 35:148-153. [PubMed: 17325983]

23. Choudat D, Frisch C, Barrat G, el Kholti A, Conso F. Occupational Exposure to Amorphous Silica Dust and Pulmonary Function. Br. J. Ind. Med. 1990; 47:763-766. [PubMed: 2173947] 
24. Pokhrel S, Nel AE, Maedler L. Custom-Designed Nanomaterial Libraries for Testing Metal Oxide Toxicity. Acc. Chem. Res. 2013; 46:632-641. [PubMed: 23194152]

25. Nel AE, Maedler L, Velegol D, Xia T, Hoek EMV, Somasundaran P, Klaessig F, Castranova V, Thompson M. Understanding Biophysicochemical Interactions at the Nano-Bio Interface. Nat. Mater. 2009; 8:543-557. [PubMed: 19525947]

26. Li R, Ji Z, Chang CH, Dunphy DR, Cai X, Meng H, Zhang H, Sun B, Wang X, Dong J, Lin S, Wang M, Liao Y-P, Brinker CJ, Nel A, Xia T. Surface Interactions with Compartmentalized Cellular Phosphates Explain Rare Earth Oxide Nanoparticle Hazard and Provide Opportunities for Safer Design. ACS Nano. 2014; 8:1771-1783. [PubMed: 24417322]

27. Liu X. Inflammatory Cytokines Augments TGF-Beta1-Induced Epithelial-Mesenchymal Transition in A549 Cells by Up-Regulating TbetaR-I. Cell Motil. Cytoskeleton. 2008; 65:935-944. [PubMed: 18792103]

28. Bonner JC. Mesenchymal Cell Survival in Airway and Interstitial Pulmonary Fibrosis. Fibrogenesis Tissue Repair. 2010; 3:1755-1536.

29. Li R, Wang X, Ji Z, Sun B, Zhang H, Chang CH, Lin S, Meng H, Liao Y-P, Wang M, Li Z, Hwang AA, Song T-B, Xu R, Yang Y, Zink JI, Nel AE, Xia T. Surface Charge and Cellular Processing of Covalently Functionalized Multiwall Carbon Nanotubes Determine Pulmonary Toxicity. ACS Nano. 2013; 7:2352-2368. [PubMed: 23414138]

30. Wang X, Duch MC, Mansukhani N, Ji Z, Liao Y-P, Wang M, Zhang H, Sun B, Chang CH, Li R, Lin S, Meng H, Xia T, Hersam MC, Nel AE. Use of a Pro-Fibrogenic Mechanism-Based Predictive Toxicological Approach for Tiered Testing and Decision Analysis of Carbonaceous Nanomaterials. ACS Nano. 2015; 9:3032-3043. [PubMed: 25646681]

31. Gregoratto D, Bailey MR, Marsh JW. Modelling Particle Retention in the Alveolar-Interstitial Region of the Human Lungs. J. Radiol. Prot. 2010; 30:491-512. [PubMed: 20826887]

32. Fruijtier-Pölloth C. The Toxicological Mode of Action and the Safety of Synthetic Amorphous Silica-A Nanostructured Material. Toxicology. 2012; 294:61-79. [PubMed: 22349641]

33. Paik U, Kim JP, Jung YS, Jung YG, Katoh T, Park JG, Hackley VA. The effect of Si dissolution on the stability of silica particles and its influence on chemical mechanical polishing for interlayer dielectrics. J. Korean Phys. Soc. 2001; 39:S201-S204.

34. He Q, Zhang Z, Gao Y, Shi J, Li Y. Intracellular Localization and Cytotoxicity of Spherical Mesoporous Silica Nano- and Microparticles. Small. 2009; 5:2722-2729. [PubMed: 19780070]

35. Ji Z, Wang X, Zhang H, Lin S, Meng H, Sun B, George S, Xia T, Nel A, Zink J. Designed Synthesis of $\mathrm{CeO}_{2}$ Nanorods and Nanowires for Studying Toxicological Effects of High Aspect Ratio Nanomaterials. ACS Nano. 2012; 6:5366-5380. [PubMed: 22564147]

36. Sun B, Ji Z, Liao Y-P, Wang M, Wang X, Dong J, Chang CH, Li R, Zhang H, Nel AE, Xia T. Engineering an Effective Immune Adjuvant by Designed Control of Shape and Crystallinity of Aluminum Oxyhydroxide Nanoparticles. ACS Nano. 2013; 7:10834-10849. [PubMed: 24261790]

37. Sun B, Wang X, Ji Z, Wang M, Liao Y-P, Chang CH, Li R, Zhang H, Nel AE, Xia T. NADPH Oxidase-Dependent NLRP3 Inflammasome Activation and its Important Role in Lung Fibrosis by Multiwalled Carbon Nanotubes. Small (Weinheim an der Bergstrasse, Germany). 2015; 11:20872097.

38. Sun B, Wang X, Ji Z, Li R, Xia T. NLRP3 Inflammasome Activation Induced by Engineered Nanomaterials. Small. 2013; 9:1595-1607. [PubMed: 23180683]

39. Dove PM, Han N, Wallace AF, De Yoreo JJ. Kinetics of Amorphous Silica Dissolution and the Paradox of the Silica Polymorphs. Proc. Natl. Acad. Sci. U. S. A. 2008; 105:9903-9908. [PubMed: 18632576]

40. Iler, RK. The Chemistry of Silica: Solubility, Polymerization, Colloid and Surface Properties and Biochemistry of Silica. Wiley; 1979. p. 896

41. Johnston CJ, Driscoll KE, Finkelstein JN, Baggs R, O'Reilly MA, Carter J, Gelein R, Oberdorster G. Pulmonary Chemokine and Mutagenic Responses in Rats after Subchronic Inhalation of Amorphous and Crystalline Silica. Toxicol. Sci. 2000; 56:405-413. [PubMed: 10911000]

42. Morishige T, Yoshioka Y, Inakura H, Tanabe A, Yao X, Narimatsu S, Monobe Y, Imazawa T, Tsunoda S-i, Tsutsumi Y, Mukai Y, Okada N, Nakagawa S. The Effect of Surface Modification of 
Amorphous Silica Particles on NLRP3 Inflammasome Mediated IL-1 Beta Production, ROS Production and Endosomal Rupture. Biomaterials. 2010; 31:6833-6842. [PubMed: 20561679]

43. Kusaka T, Nakayama M, Nakamura K, Ishimiya M, Furusawa E, Ogasawara K. Effect of Silica Particle Size on Macrophage Inflammatory Responses. PLoS One. 2014; 9

44. van der Zande M, Vandebriel R, Groot M, Kramer E, Herrera Rivera Z, Rasmussen K, Ossenkoppele J, Tromp P, Gremmer E, Peters R, Hendriksen P, Marvin H, Hoogenboom R, Peijnenburg A, Bouwmeester H. Sub-Chronic Toxicity Study in Rats Orally Exposed to Nanostructured Silica. Part. Fibre Toxicol. 2014; 11:8. [PubMed: 24507464]

45. Kim KD, Pokhrel S, Wang Z, Ling H, Zhou C, Liu Z, Hunger M, Mädler L, Huang J. Tailoring High-Performance Pd Catalysts for Chemoselective Hydrogenation Reactions via Optimizing the Parameters of the Double-Flame Spray Pyrolysis. ACS Catal. 2016; 6:2372-2381.

46. Wang Z, Pokhrel S, Chen M, Hunger M, Mädler L, Huang J. Palladium-Doped Silica-Alumina Catalysts Obtained from Double-Flame FSP for Chemoselective Hydrogenation of the Model Aromatic Ketone Acetophenone. J. Catal. 2013; 302:10-19.

47. Joshi S, Ghosh I, Pokhrel S, Mädler L, Nau WM. Interactions of Amino Acids and Polypeptides with Metal Oxide Nanoparticles Probed by Fluorescent Indicator Adsorption and Displacement. ACS Nano. 2012; 6:5668-5679. [PubMed: 22591378]

48. Pokhrel S, Birkenstock J, Schowalter M, Rosenauer A, Mädler L. Growth of Ultrafine Single Crystalline WO3 Nanoparticles Using Flame Spray Pyrolysis. Cryst. Growth Des. 2010; 10:632639.

49. Ji Z, Jin X, George S, Xia T, Meng H, Wang X, Suarez E, Zhang H, Hoek EMV, Godwin H, Nel AE, Zink JI. Dispersion and Stability Optimization of TiO2 Nanoparticles in Cell Culture Media. Environ. Sci. Technol. 2010; 44:7309-7314. [PubMed: 20536146]

50. Liu X, Situ A, Kang Y, Villabroza KR, Liao Y, Chang CH, Donahue T, Nel AE, Meng H. Irinotecan Delivery by Lipid-Coated Mesoporous Silica Nanoparticles Shows Improved Efficacy and Safety over Liposomes for Pancreatic Cancer. ACS Nano. 2016; 10:2702-2715. [PubMed: 26835979]

51. Wang X, Xia T, Addo Ntim S, Ji Z, Lin S, Meng H, Chung C-H, George S, Zhang H, Wang M, Li N, Yang Y, Castranova V, Mitra S, Bonner JC, Nel AE. Dispersal State of Multiwalled Carbon Nanotubes Elicits Profibrogenic Cellular Responses That Correlate with Fibrogenesis Biomarkers and Fibrosis in the Murine Lung. ACS Nano. 2011; 5:9772-9787. [PubMed: 22047207]

52. Shvedova AA, Kisin E, Murray AR, Johnson VJ, Gorelik O, Arepalli S, Hubbs AF, Mercer RR, Keohavong P, Sussman N, Jin J, Yin J, Stone S, Chen BT, Deye G, Maynard A, Castranova V, Baron PA, Kagan VE, Inhalation vs. Aspiration of Single-Walled Carbon Nanotubes in C57BL/6 Mice: Inflammation, Fibrosis, Oxidative Stress, and Mutagenesis. Am. J. Physiol.: Lung Cell. Mol. Physiol. 2008; 295:L552-L565. [PubMed: 18658273]

53. Hao M, Comier S, Wang M, Lee JJ, Nel A. Diesel Exhaust Particles Exert Acute Effects on Airway Inflammation and Function in Murine Allergen Provocation Models. J. Allergy Clin. Immunol. 2003; 112:905-914. [PubMed: 14610479]

54. Wang X, Ji Z, Chang CH, Zhang H, Wang M, Liao Y-P, Lin S, Meng H, Li R, Sun B, Winkle LV, Pinkerton KE, Zink JI, Xia T, Nel AE. Use of Coated Silver Nanoparticles to Understand the Relationship of Particle Dissolution and Bioavailability to Cell and Lung Toxicological Potential. Small. 2014; 10:385-398. [PubMed: 24039004]

55. Zhang H, Ji Z, Xia T, Meng H, Low-Kam C, Liu R, Pokhrel S, Lin S, Wang X, Liao Y-P, Wang M, Li L, Rallo R, Damoiseaux R, Telesca D, Mädler L, Cohen Y, Zink JI, Nel AE. Use of Metal Oxide Nanoparticle Band Gap To Develop a Predictive Paradigm for Oxidative Stress and Acute Pulmonary Inflammation. ACS Nano. 2012; 6:4349-4368. [PubMed: 22502734] 
A

A

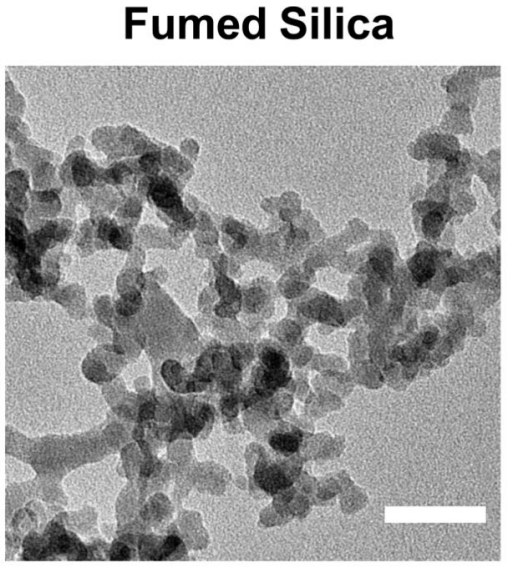

Fumed Silica

7\% Ti Fumed Silica

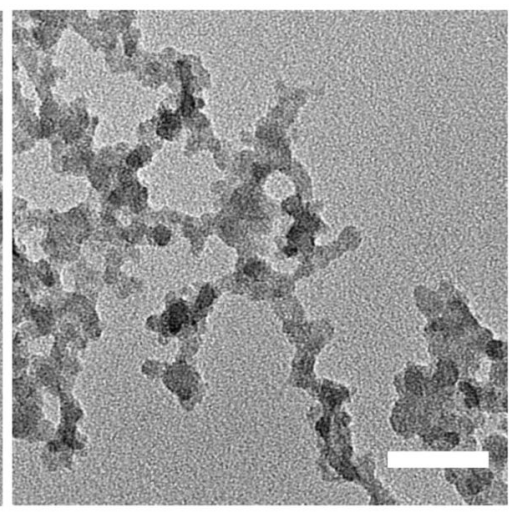

B

Hydrodynamic size and $\xi$-potential of non-doped and $7 \%$ Ti-doped fumed silica and their silanol concentration

\begin{tabular}{|c|c|c|c|c|c|}
\hline & \multicolumn{2}{|c|}{ hydrodynamic size (nm) } & \multicolumn{2}{|c|}{$\zeta$-potential (mV) } & \multirow[b]{2}{*}{ Total silanol $\left(\mathrm{OH} / \mathrm{nm}^{2}\right)$} \\
\hline & Water & $\begin{array}{c}\text { PBS @ BSA \& } \\
\text { DPPC }\end{array}$ & Water & $\begin{array}{c}\text { PBS @ BSA \& } \\
\text { DPPC }\end{array}$ & \\
\hline Fumed silica & $221.5 \pm 5.8$ & $210.9 \pm 16.1$ & $-35.2 \pm 1.4$ & $-12.3 \pm 4.6$ & $12.86 \pm 1.87$ \\
\hline 7\% Ti Fumed silica & $252.1 \pm 20.5$ & $155.3 \pm 19.9$ & $-33.9 \pm 1.5$ & $-15.6 \pm 3.4$ & $7.63 \pm 2.38$ \\
\hline
\end{tabular}

Figure 1. Characterization of fumed silica nanoparticles

(A) Representative TEM images of pristine and 7\% Ti-doped fumed silica nanoparticles. The images were taken with a JEOL 1200 EX TEM with an accelerating voltage of $80 \mathrm{kV}$. The scale bar is $50 \mathrm{~nm}$. (B) Hydrodynamic size and $\xi$-potential of pristine and 7\% Ti-doped fumed silica nanoparticles in exposure media, and their silanol concentration calculated through the integration of the FTIR band data at $4500 \mathrm{~cm}^{-1}$. 
A

FS $1^{\text {st }}$ dose

FS $2^{\text {nd }}$ dose

FS $3^{\text {rd }}$ dose

(1x2

(2x2

$13 \times 2$

$1 \times 3$

$2 \times 3$

$3 \times 3$

1x7)

2x7)

$3 \times 7$ )

Repetitive dose

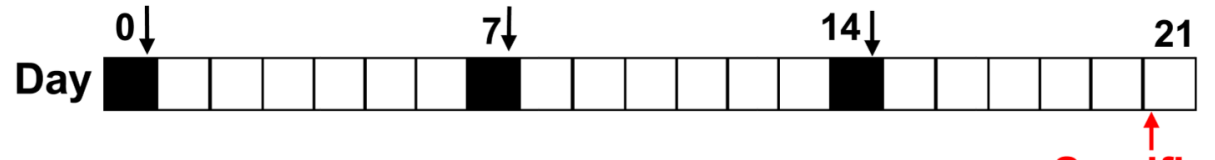

Cons?

Intratracheal

FS $1 \times 6,1 \times 9$

Sacrifice

Instillation

Single dose or $1 \times 21$

@ 21D

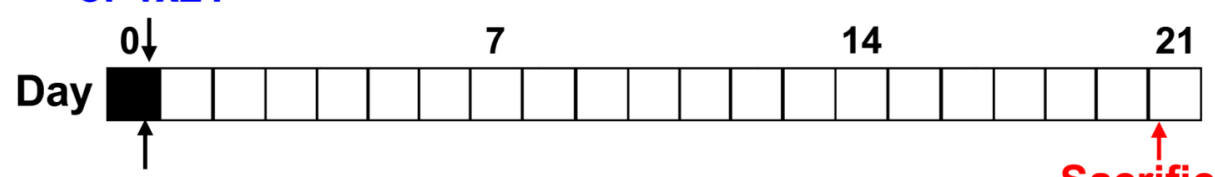

PBS, QTZ 
B

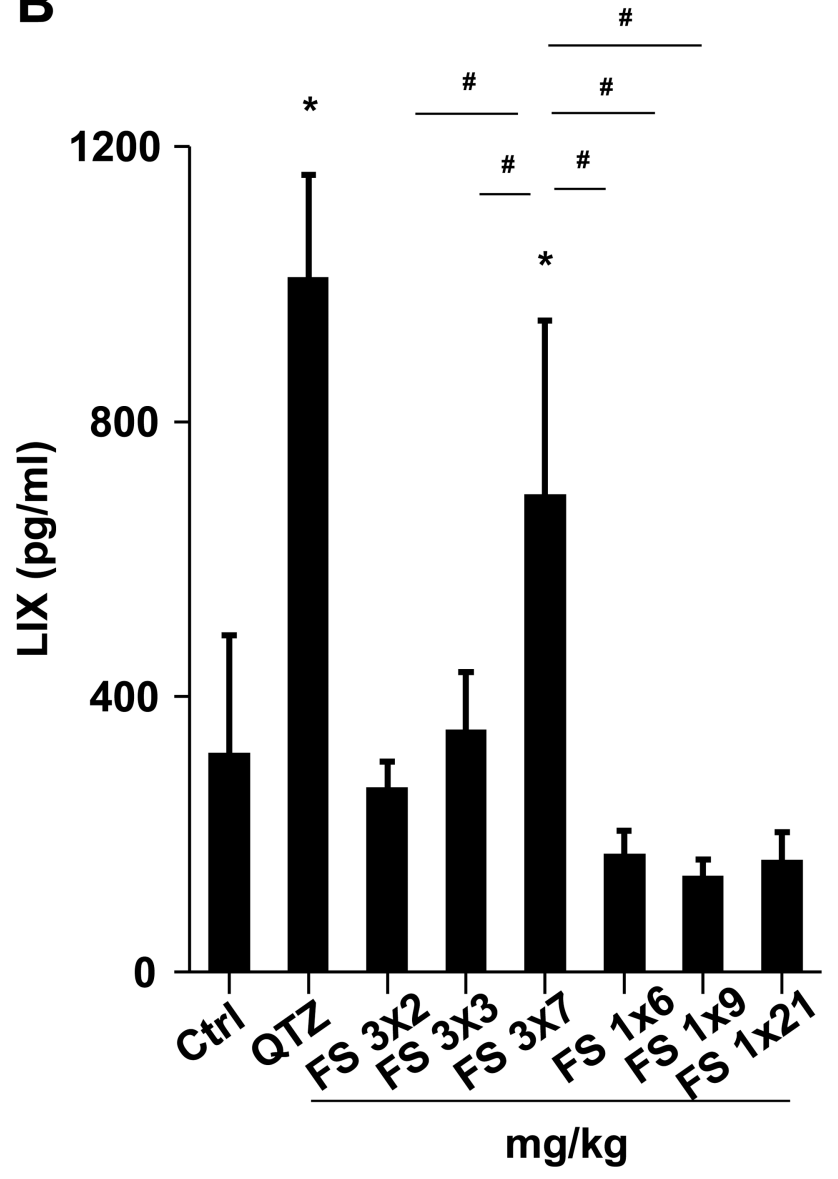

C

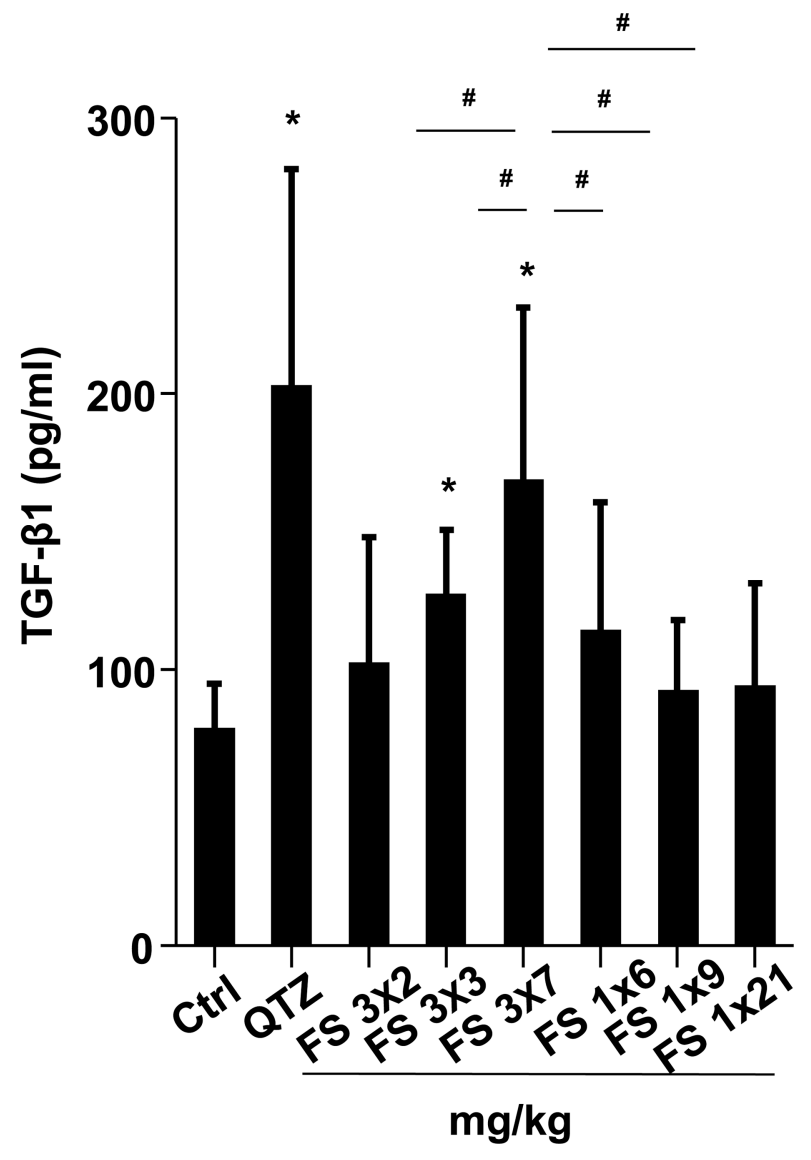

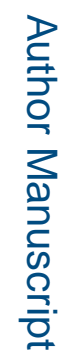

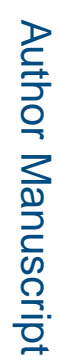


$\mathbf{D}$

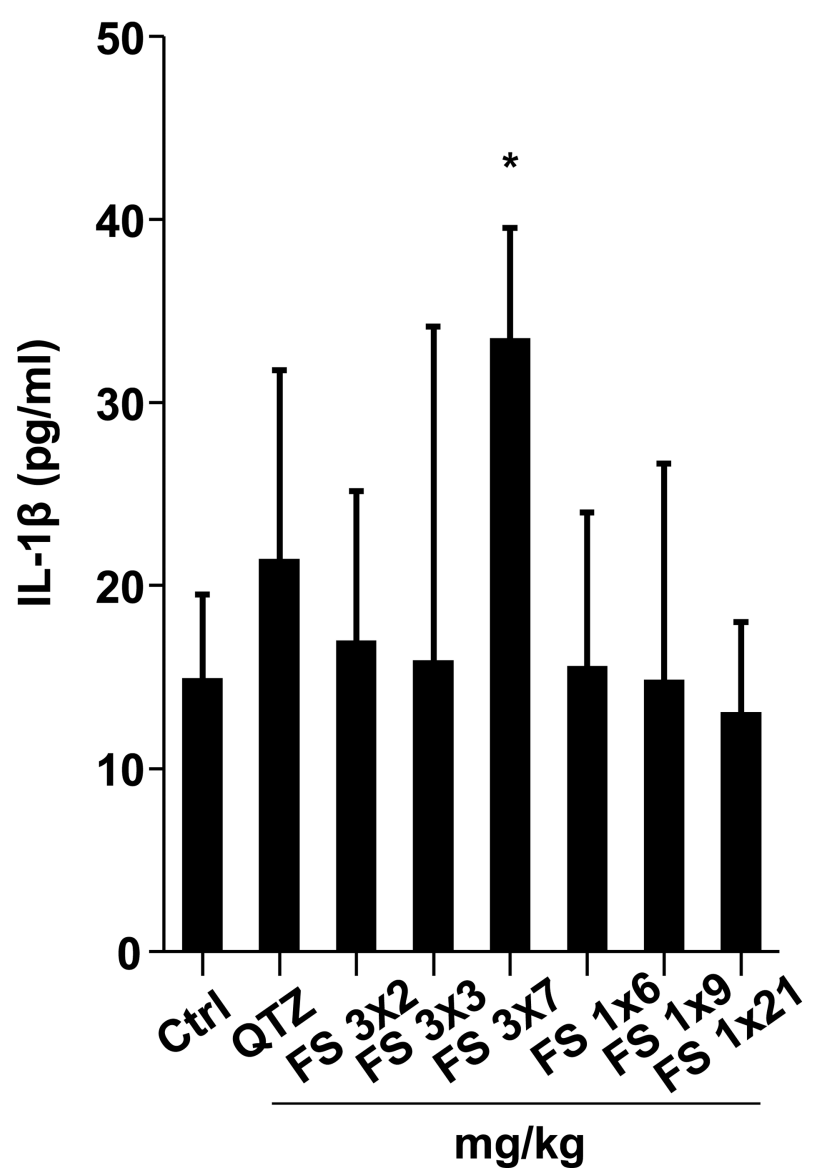

$\mathbf{E}$

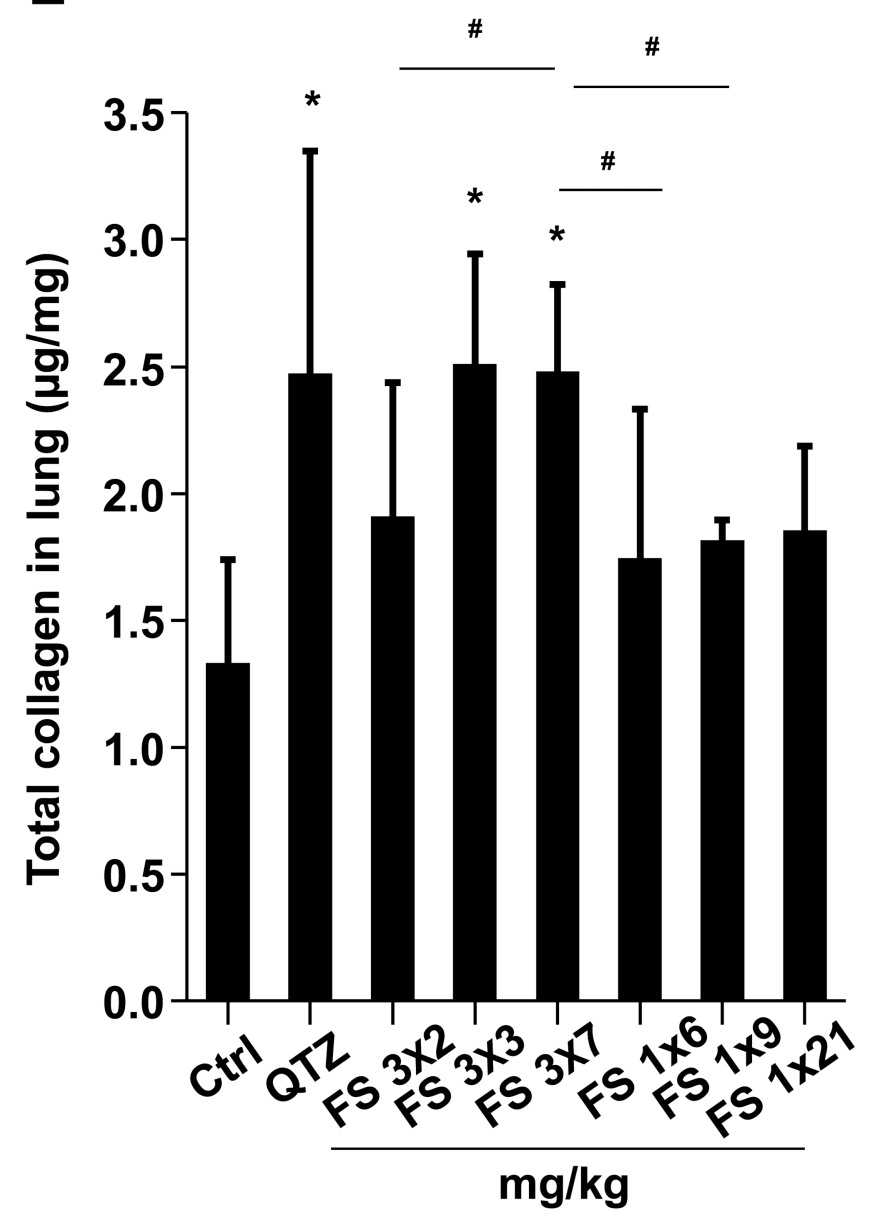


Ctrl

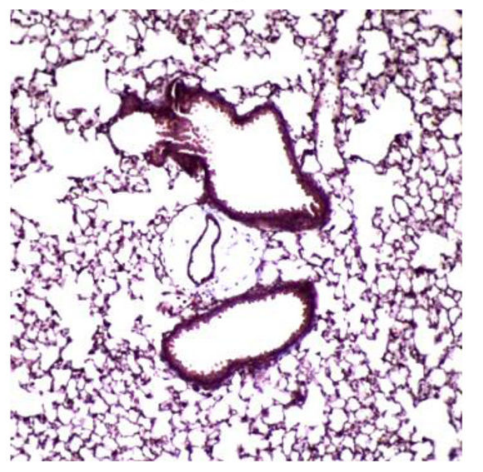

QTZ

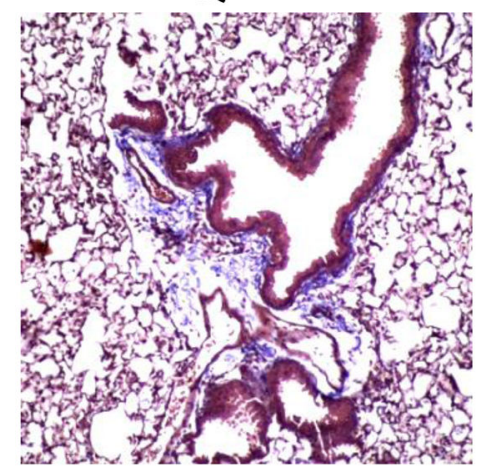

\section{$3 \times 2 \mathrm{mg} / \mathrm{kg}$} $3 \times 3 \mathrm{mg} / \mathrm{kg}$ $3 \times 7 \mathrm{mg} / \mathrm{kg}$
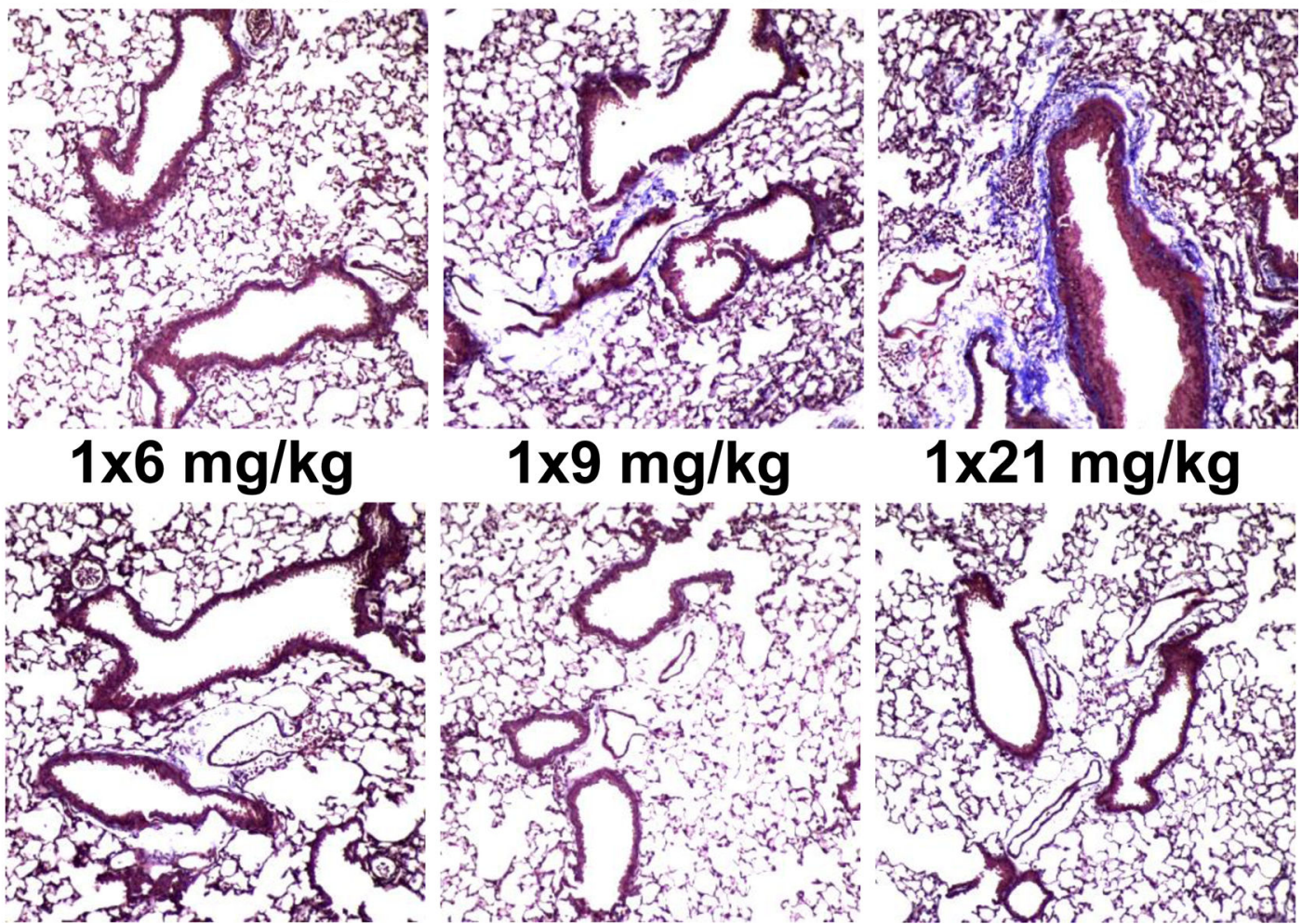

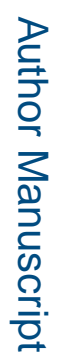




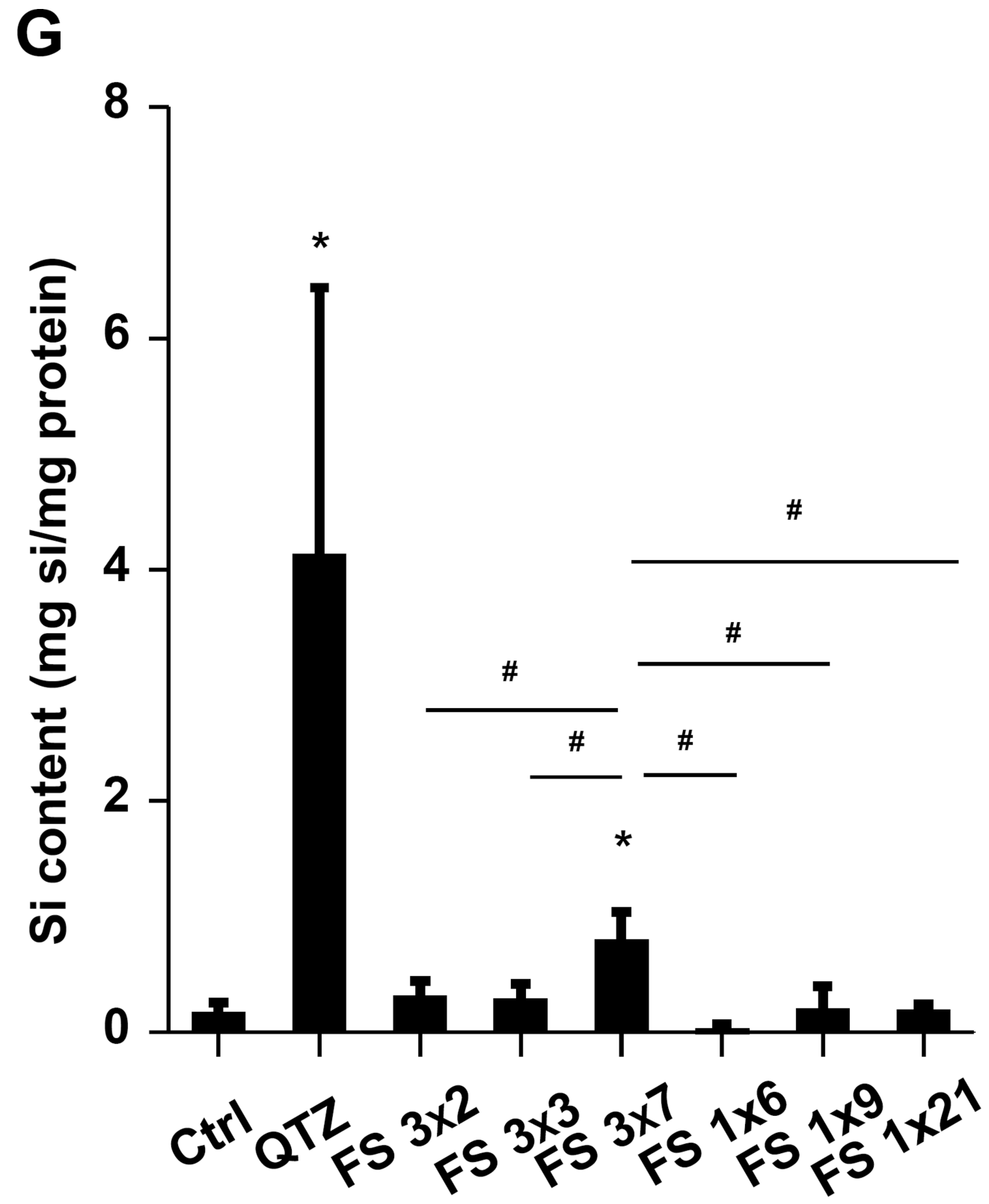

\section{$\mathrm{mg} / \mathrm{kg}$}

Figure 2. Assessment of sub-chronic pulmonary effects of fumed silica by single vs. repetitive exposures in murine lung

(A) Schematic presentation of the repetitive exposure conditions in the murine lung. C57BL/6 ( $\mathrm{n}=6)$ mice were exposed to either 3 doses of 2,3 , and $7 \mathrm{mg} / \mathrm{kg}$, one week apart (i.e., $3 \times 2,3 \times 3$, and $3 \times 7 \mathrm{mg} / \mathrm{kg}$ ) compared to single dose of 6,9 , and $21 \mathrm{mg} / \mathrm{kg}$ by oropharyngeal aspiration. (B-D) On day 21, BAL fluid was collected to determine (B) LIX, (C) TGF- $\beta 1$, and (D) IL-1 $\beta$ levels. MIN-U-SIL, a natural form of a-quartz (QTZ), was used as a positive control. (E) Total collagen content in murine lung was determined by the Sircol collagen assay. (F) Masson's Trichrome staining was used to visualize collagen deposition in 
the lungs of animals treated with fumed silica. Collagen is stained blue in the micrographs. (G) ICP-OES analysis showing silicon abundance in the lung. ${ }^{*} p<0.05$ compared to control mice. ${ }^{\#} p<0.05$ compared to mice treated with 3 doses of $7 \mathrm{mg} / \mathrm{kg}$ fumed silica. 
Fumed silica dissolution

A

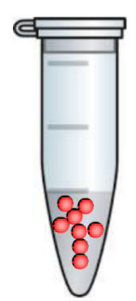

Incubate @ 37

- C for 0,6 and $40 \mathrm{~h}$

Centrifuge at

Fumed silica in

$15,000 \mathrm{rpm}$ for $1 \mathrm{~h}$

exposure media

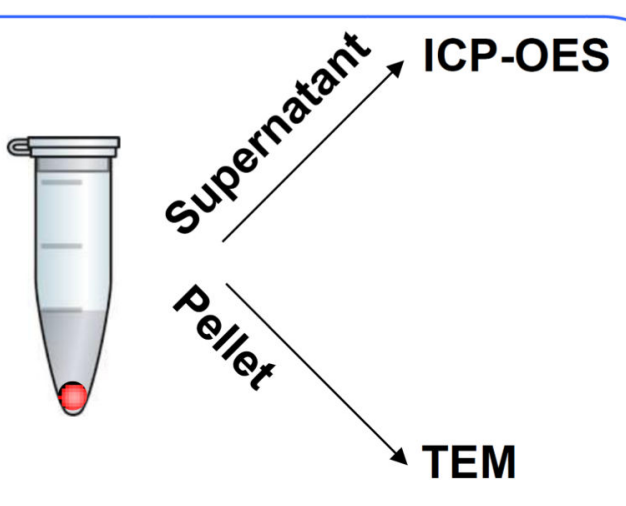

B

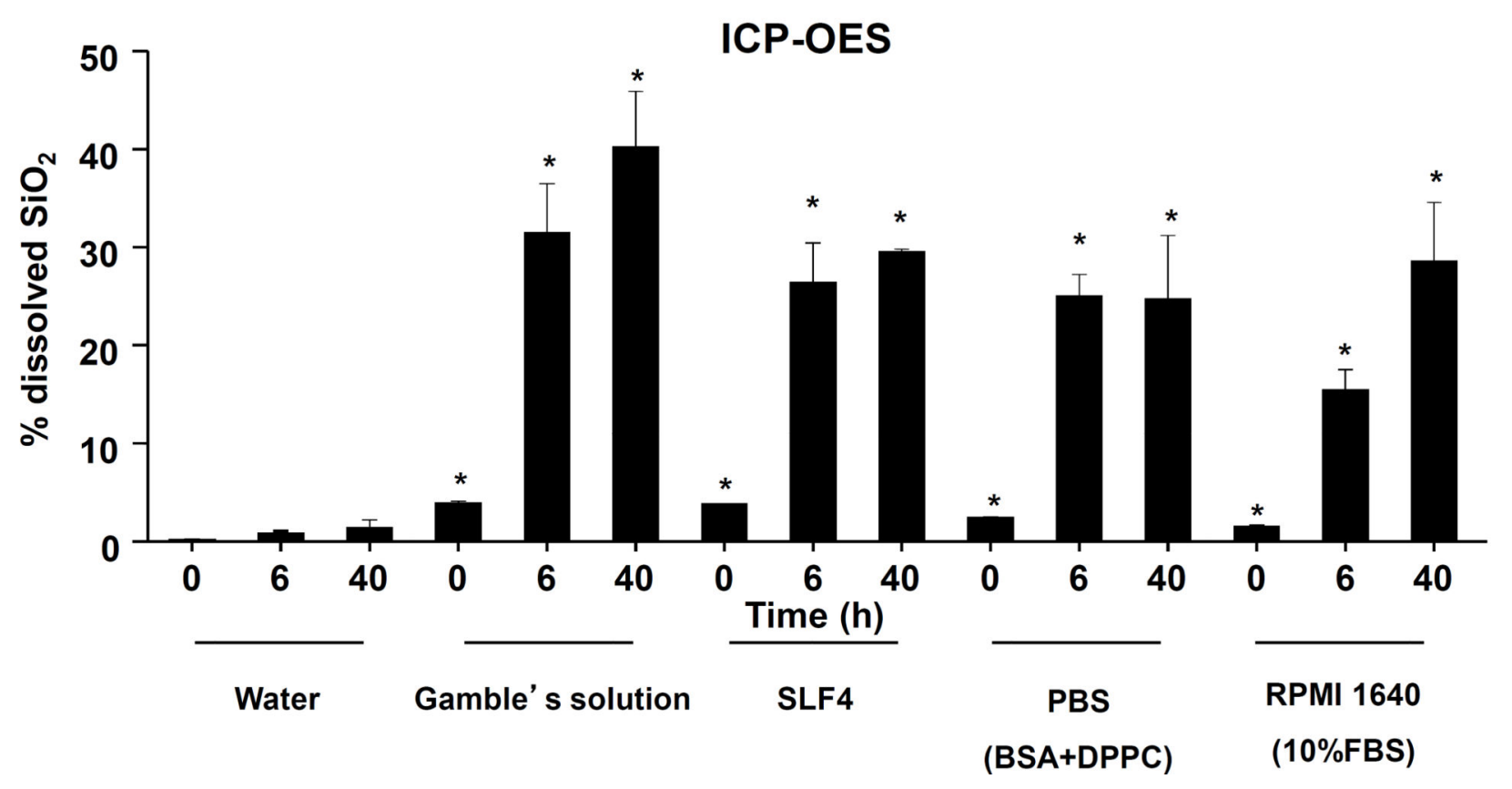

Figure 3. Fumed silica dissolution in simulated biological fluids

(A) Schematic representation of ICP-OES analysis to study Si release from fumed silica in exposure media. (B) ICP-OES analysis to study time-dependent Si release in various exposure media. $20 \mu \mathrm{L}$ of the fumed silica stock solution $(5 \mathrm{mg} / \mathrm{mL})$ were added to $980 \mu \mathrm{L}$ of exposure media before sonication. The particle suspension was incubated at $37^{\circ} \mathrm{C}$. Samples were collected at 0, 6, and $40 \mathrm{~h}$, centrifuged at 15,000 rpm, and each supernatant was collected for acid digestion and ICP-OES analysis. * $p<0.05$ compared to control exposure media without particles. 
A

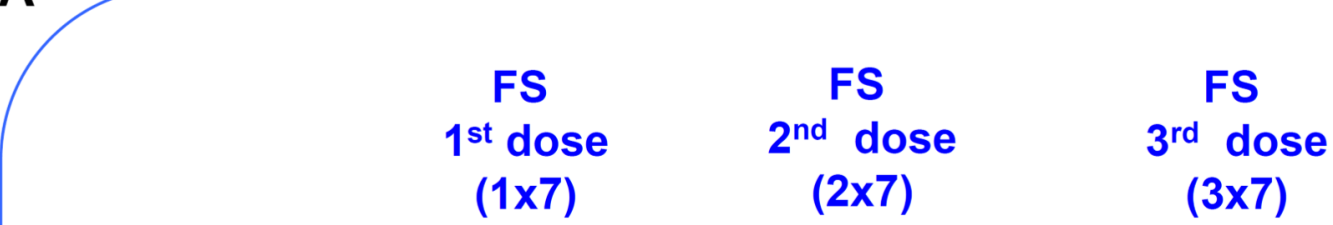

Repetitive dose

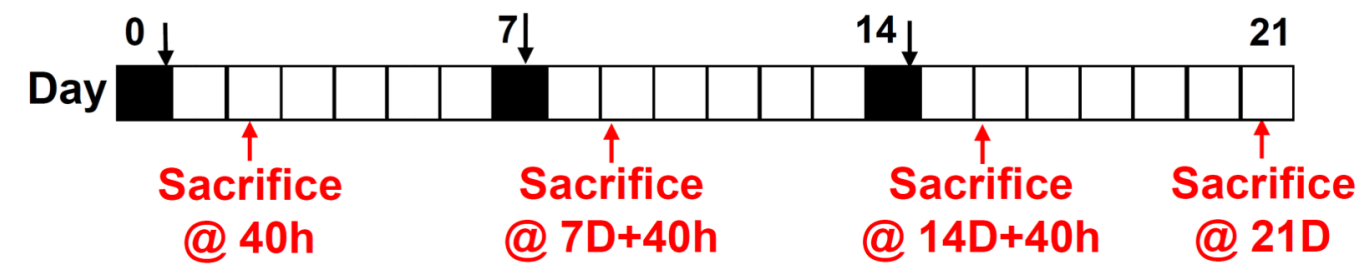

Intratracheal Instillation

FS $1 \times 21$

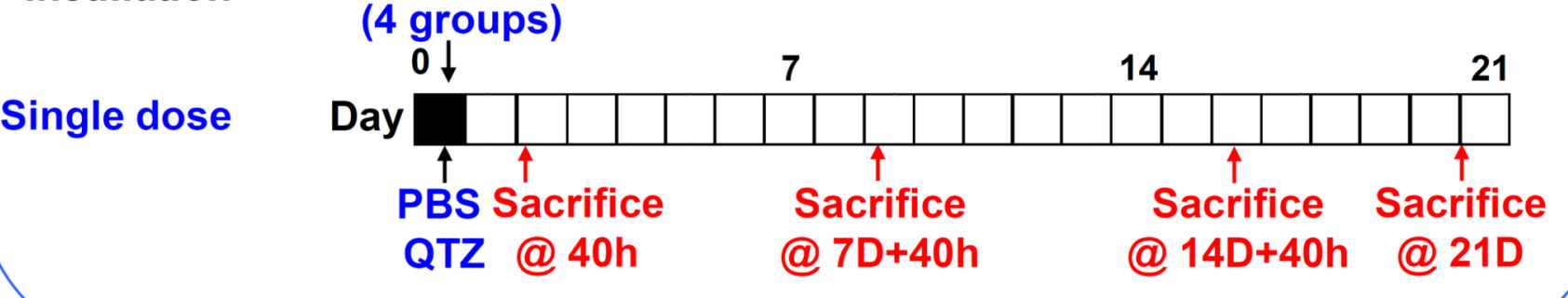


B

C

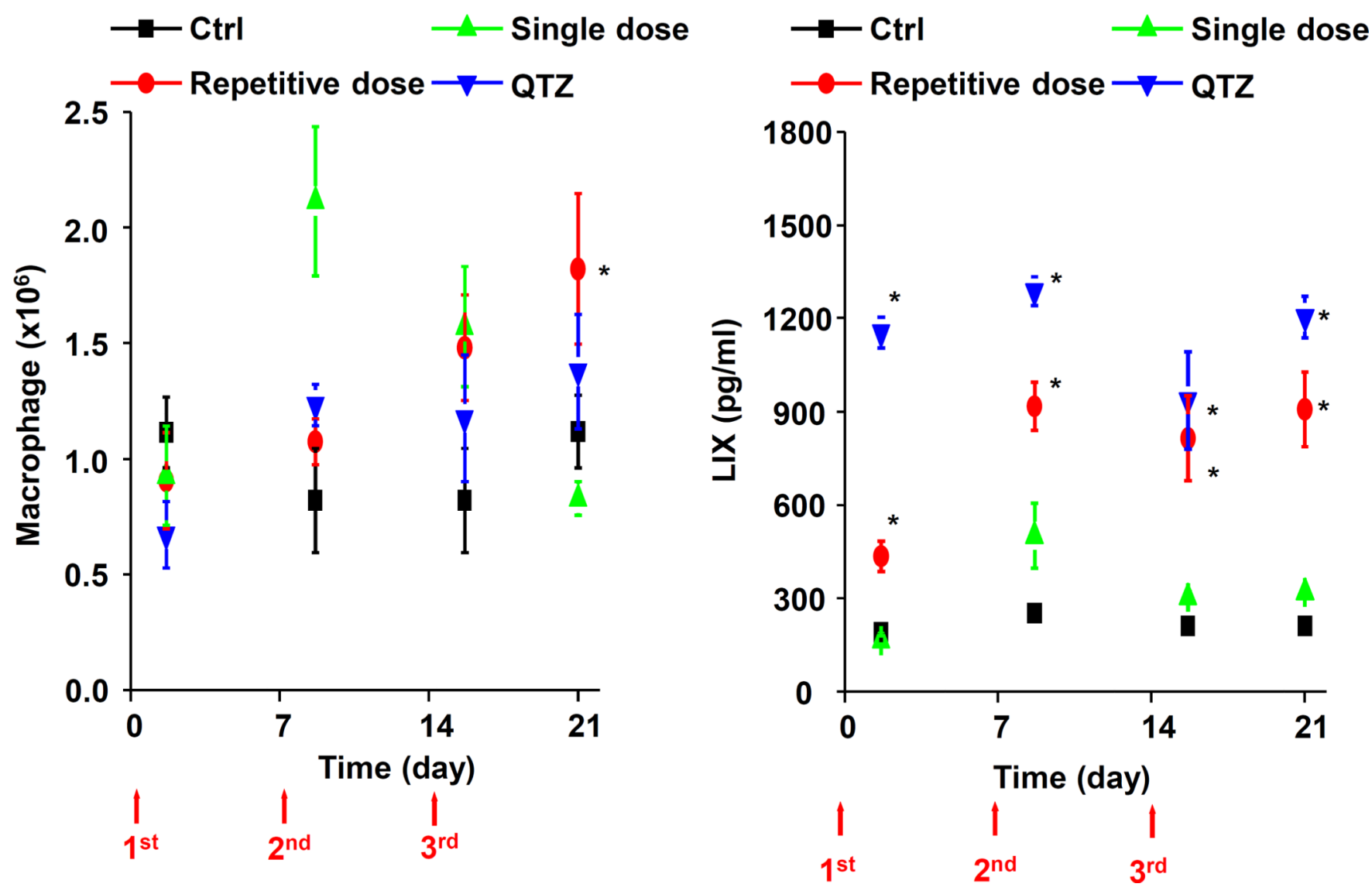


D

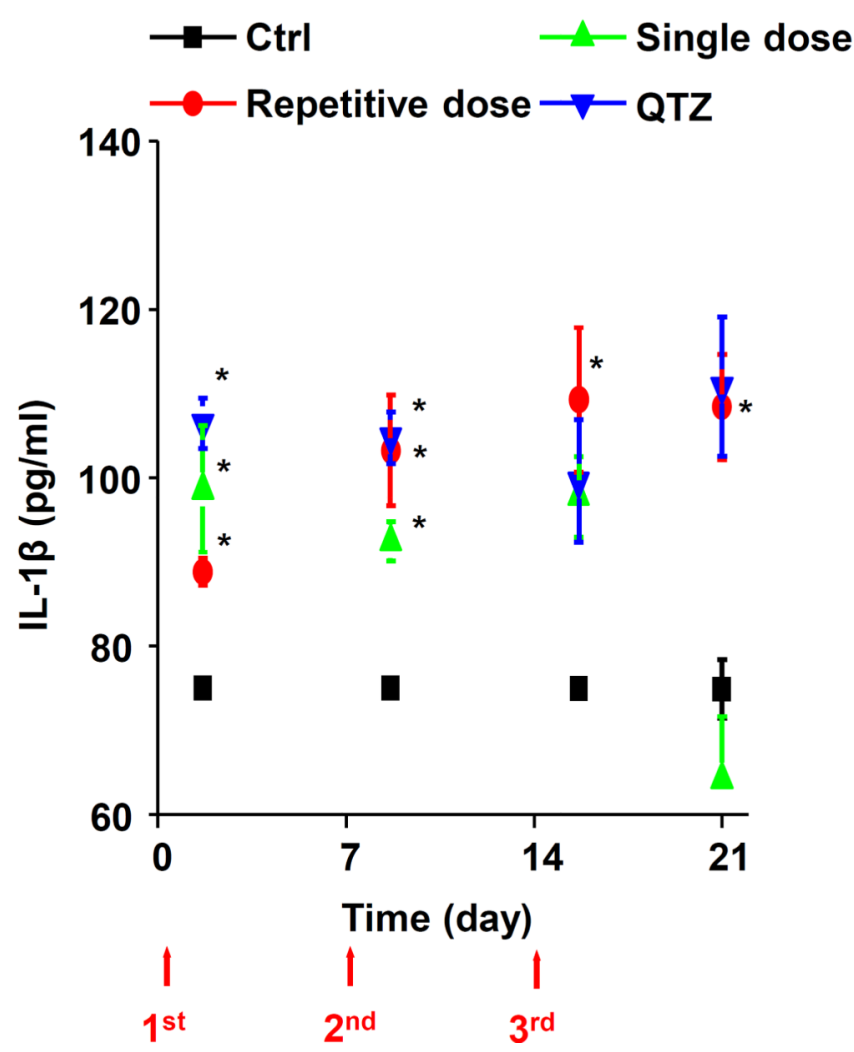

$\mathbf{E}$

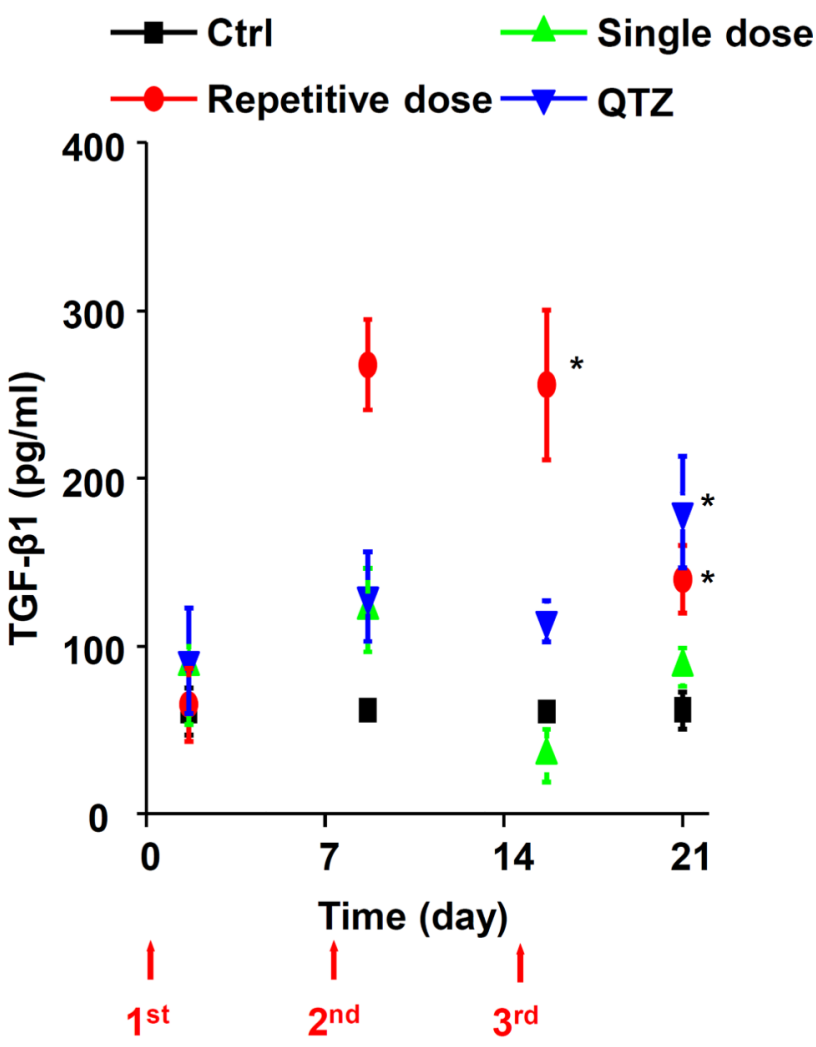

Figure 4. Comparative analysis of the cellular and cytokine kinetics of single vs. repetitive fumed silica exposures

(A) Schematic representation of the fumed silica exposure effects of $3 \times 7 \mathrm{mg} / \mathrm{kg}$ (on days 0 , 7 and 14) fumed silica compared to a single $21 \mathrm{mg} / \mathrm{kg}$ instillation on day 0 . The animals were sacrificed $40 \mathrm{~h}$ after each day of instillation as well as on day 21. (B-E) BAL fluid was collected to determine (B) macrophage counts, (C) LIX, (D) IL- $\beta$, and (E) TGF- $\beta 1$ levels. ${ }^{*} p<0.05$ compared to control mice without particle treatment. 
Trichrome Staining

Repetitive dose Single dose

A

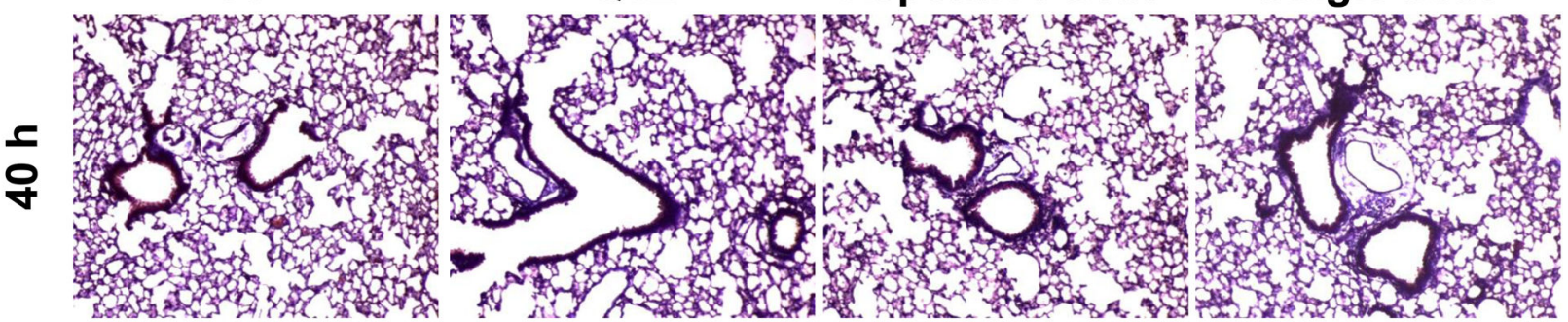

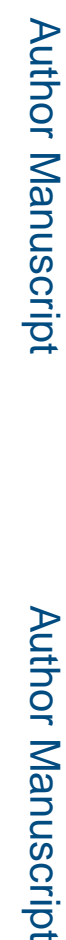
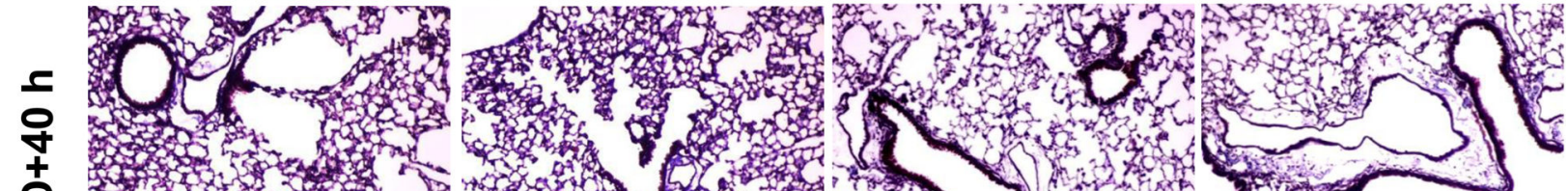

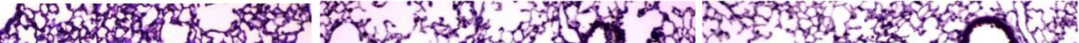

$+$

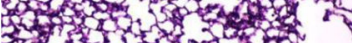

N

$\stackrel{\underline{E}}{\underline{E}}$
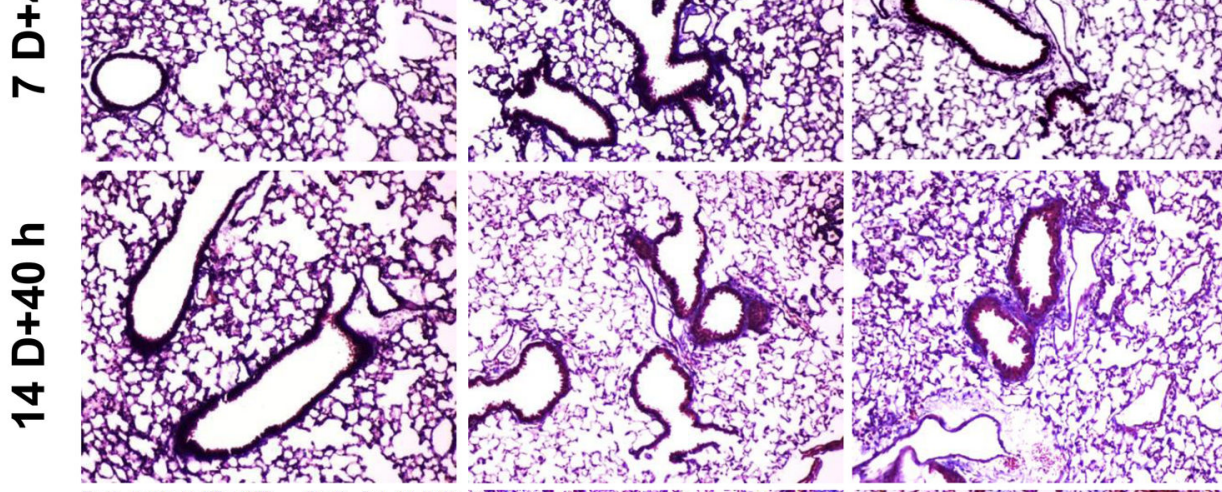

$\times 6,1$
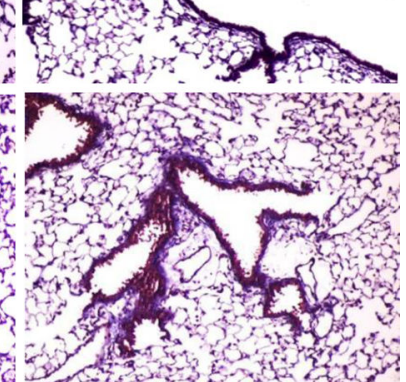

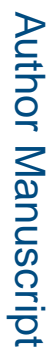
(3)

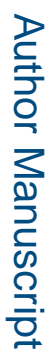


B

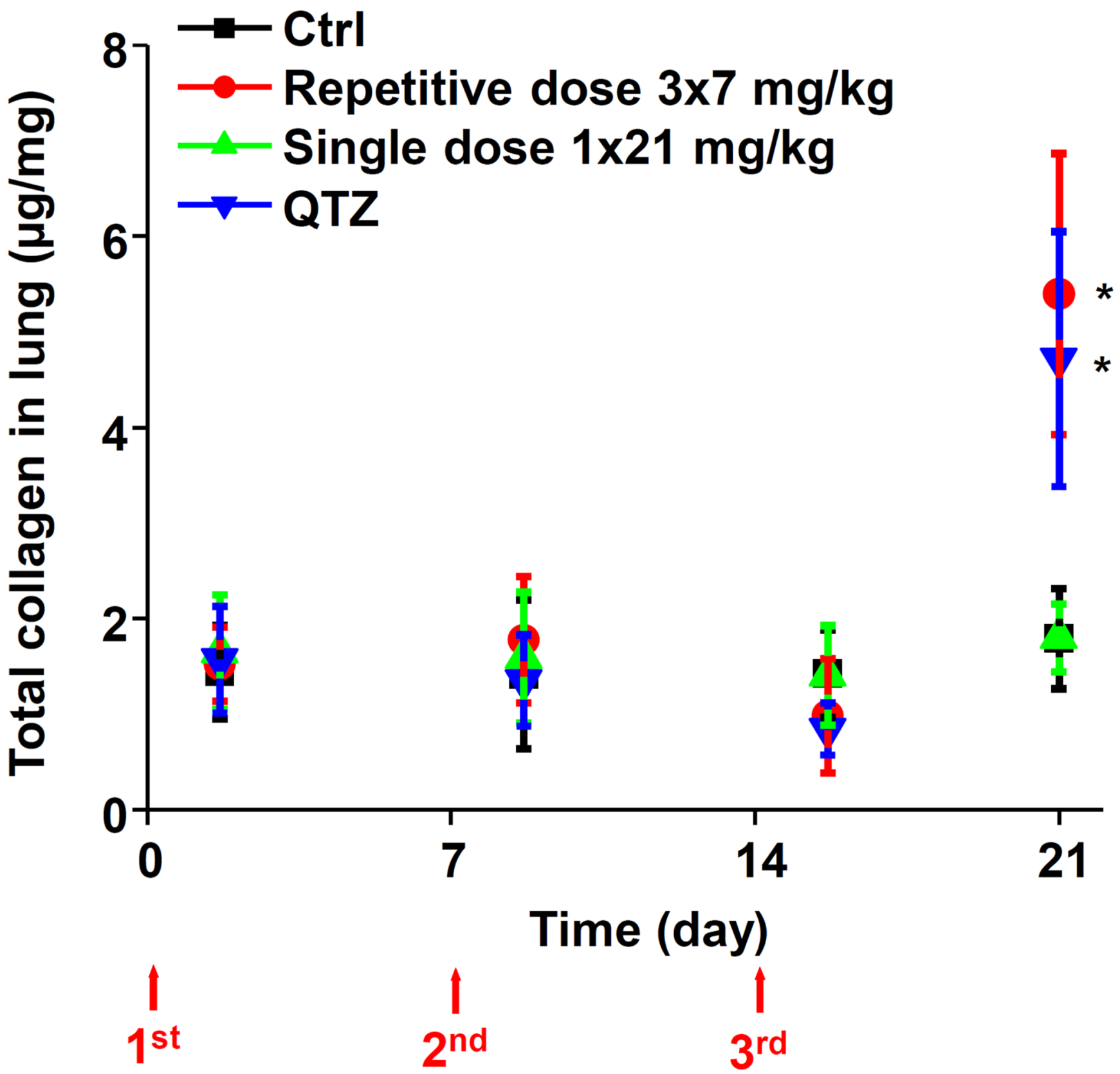

Figure 5. Time-dependent analysis of the pro-fibrogenic effects of repetitive fumed silica exposure in mice

(A) Masson's Trichrome staining was used to determine the collagen deposition in the lungs of animals treated with fumed silica. (B) Total collagen content in the murine lung, as determined by the Sircol assay. ${ }^{*} p<0.05$ compared to control mice without particle treatment. 
Fumed silica distribution (NIR imaging)

A
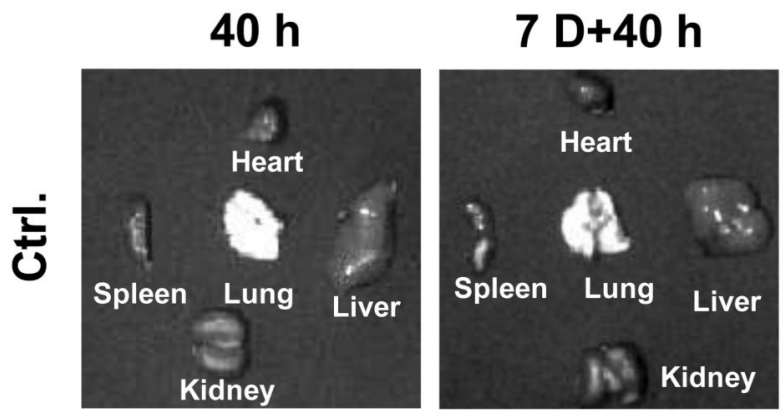

$14 \mathrm{D}+40 \mathrm{~h}$

$21 \mathrm{D}$
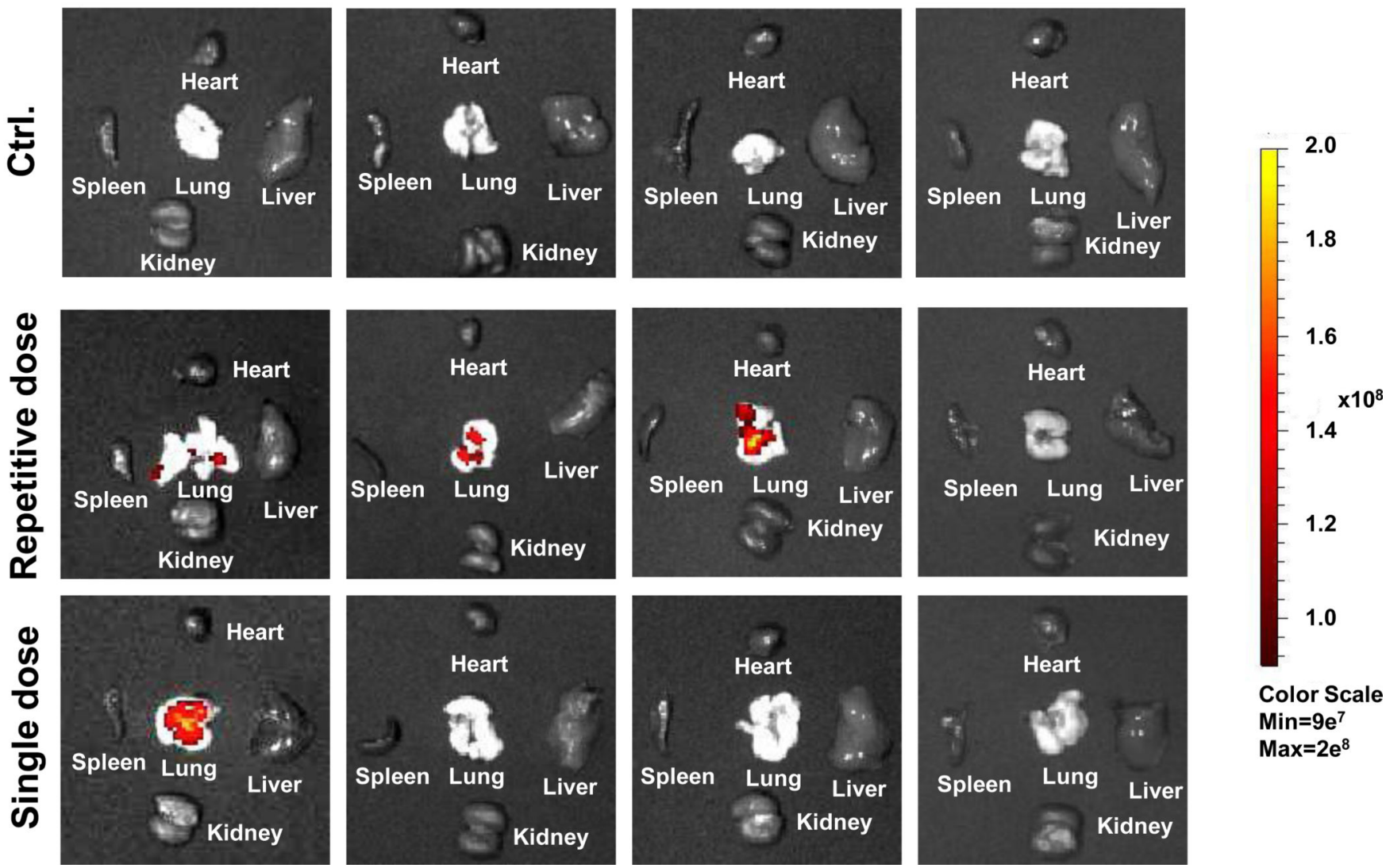

\section{Min $=9 \mathrm{e}^{7}$}

$\operatorname{Max}=2 e^{8}$ 

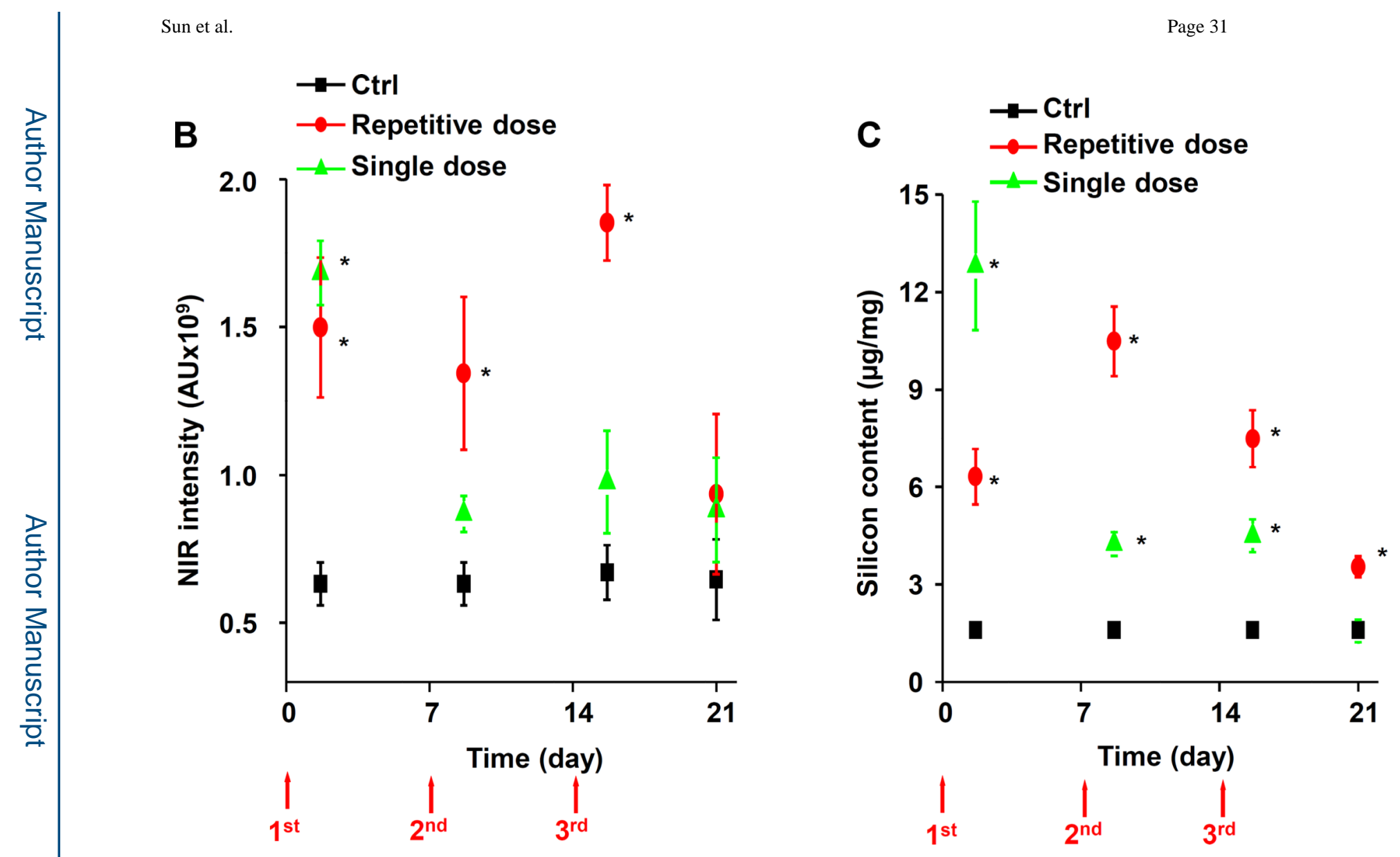


\section{Immunohistochemistry for macrophages}

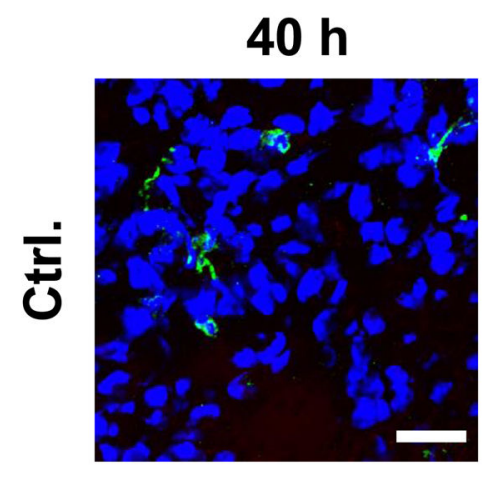

$7 \mathrm{D}+40 \mathrm{~h}$
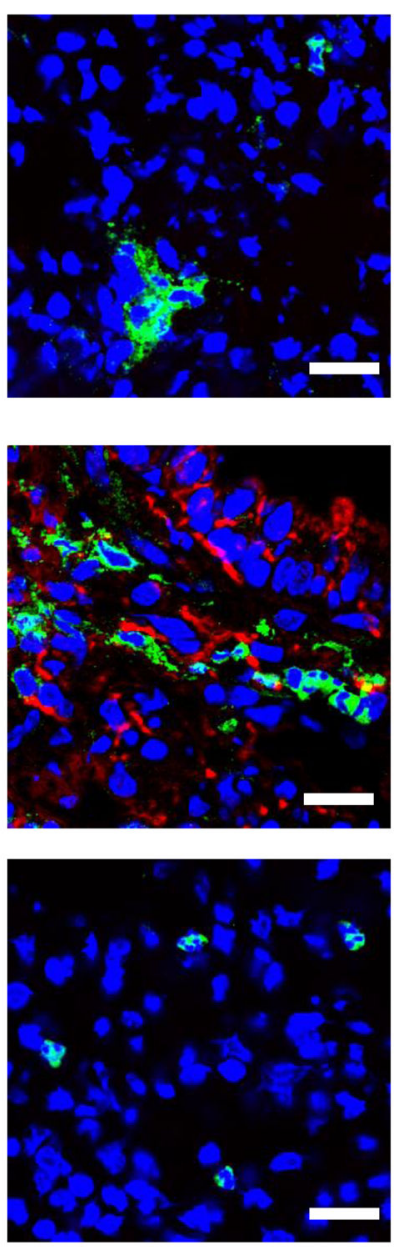

Macrophage
$14 \mathrm{D}+40 \mathrm{~h}$
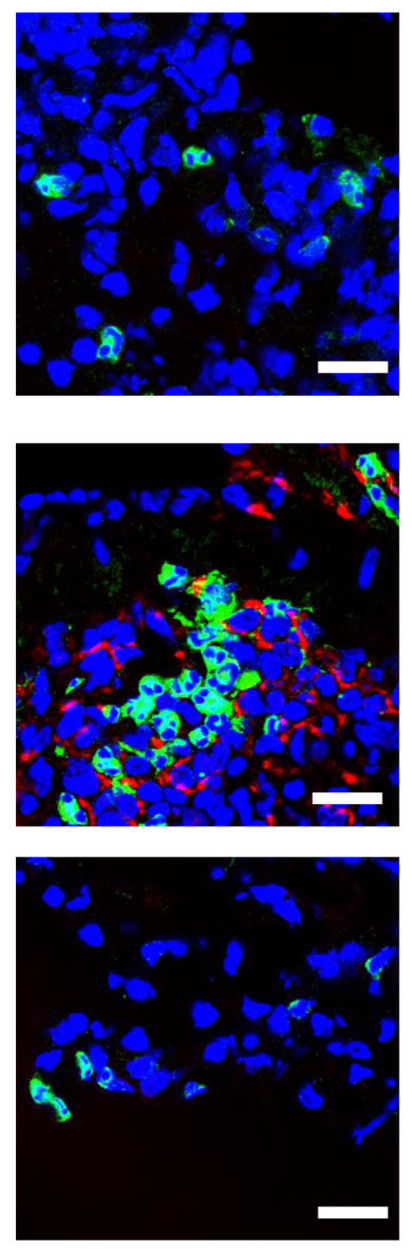

Nuclei

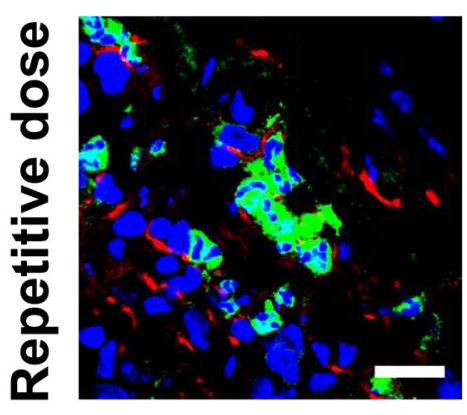

\section{Fumed silica}

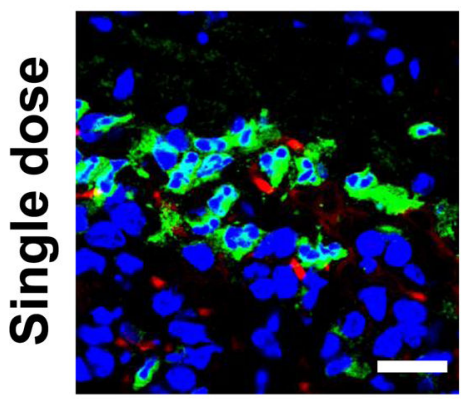

Figure 6. Fumed silica bio-distribution in the lung following oropharyngeal aspiration C57BL/6 ( $\mathrm{n}=3$ ) mice were exposed to $3 \times 7 \mathrm{mg} / \mathrm{kg}$ (on days 0,7 and 14) NIR-labeled fumed silica compared to a single $21 \mathrm{mg} / \mathrm{kg}$ instillation on day 0 . Separate groups of animals were sacrificed $40 \mathrm{~h}$ after each instillation day as well as on day 21. (A) IVIS optical imaging system was used to study the fumed silica distribution in mice. To visualize fumed silica distribution in vivo, mice were sacrificed and major organs were collected for ex vivo imaging. Representative animal images were collected at different time points. (B)

Fluorescence intensity in the operator-defined region of interest (fumed silica) was analyzed by Xenogen software and quantitatively expressed. ${ }^{*} p<0.05$ compared to control mice. (C) ICP-OES was used to quantify the Si abundance in the lung using our established procedure. ${ }^{*} p<0.05$ compared to control mice. (D) Immunohistochemistry to determine the recruitment of macrophages (green). Slices of the lung tissues from different treatment groups were used for sectioning, which were overlaid with anti-F4/80 primary antibodies 
and reacted with an Alexa Fluor 488-conjugated secondary antibody (green). Fumed silica was labeled with NIR dye (red) and cell nuclei were stained with Hoechst 33342 (blue). Slides were visualized under a Leica confocal SP2 1P-FCS microscope (Advanced Light Microscopy/Spectroscopy Shared Facility, UCLA). The scale bar is $20 \mu \mathrm{m}$. 
A

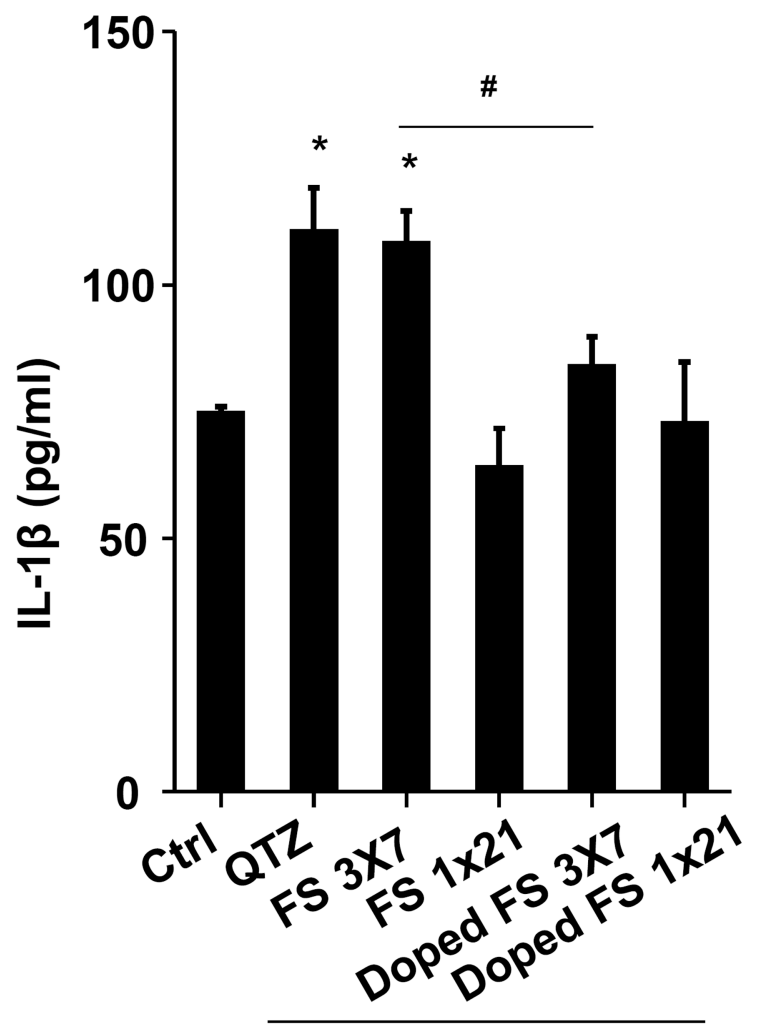

$\mathrm{mg} / \mathrm{kg}$
B

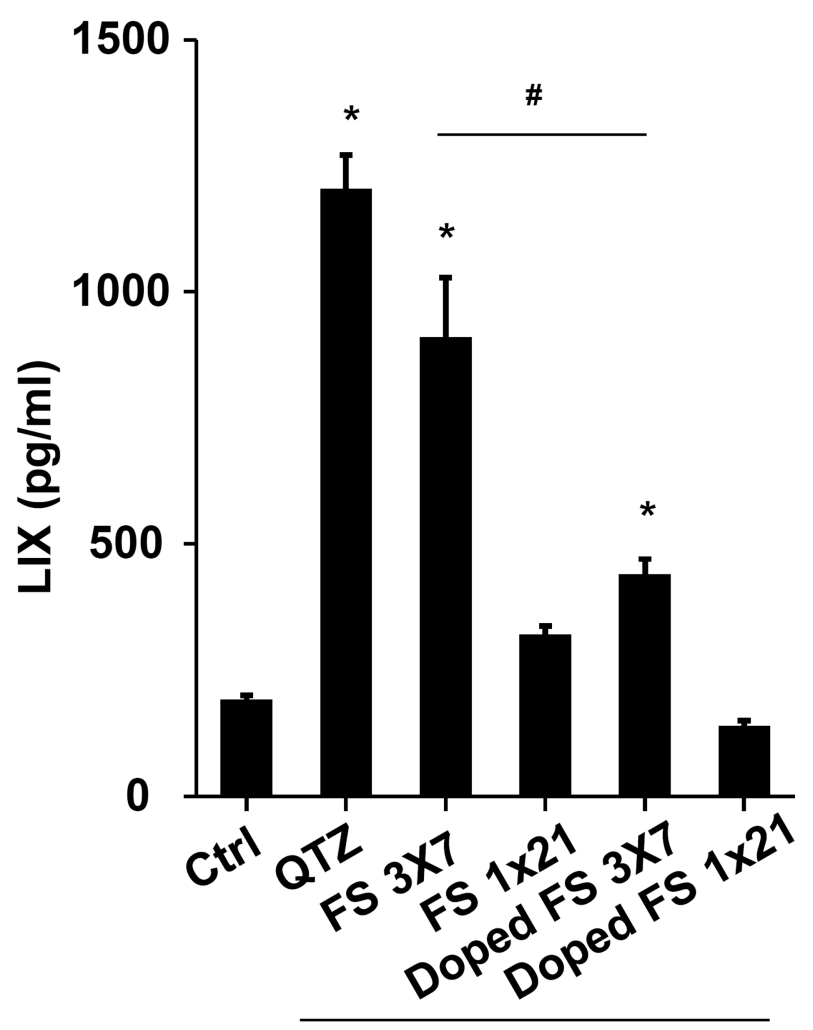

$\mathrm{mg} / \mathrm{kg}$

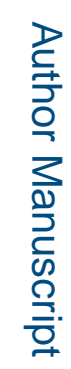


C

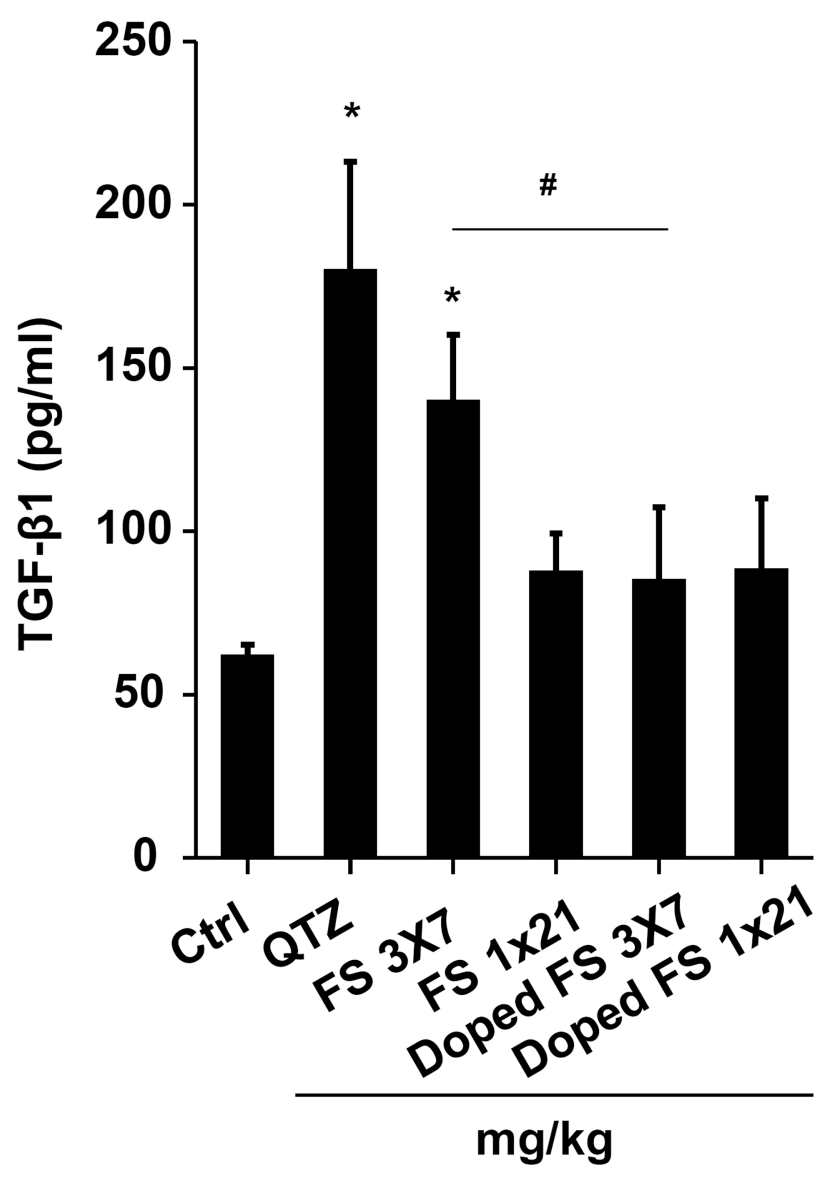

D

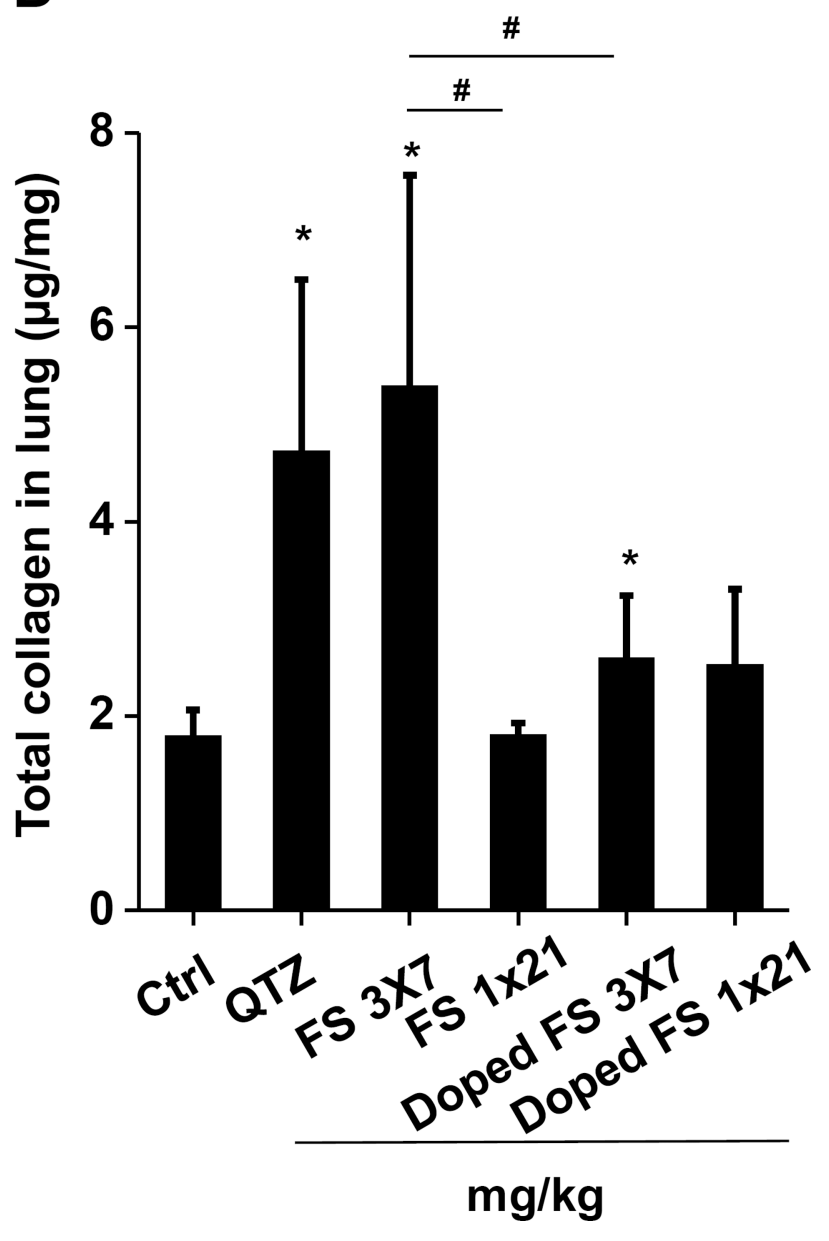

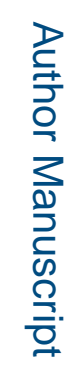




\section{Trichrome Staining}

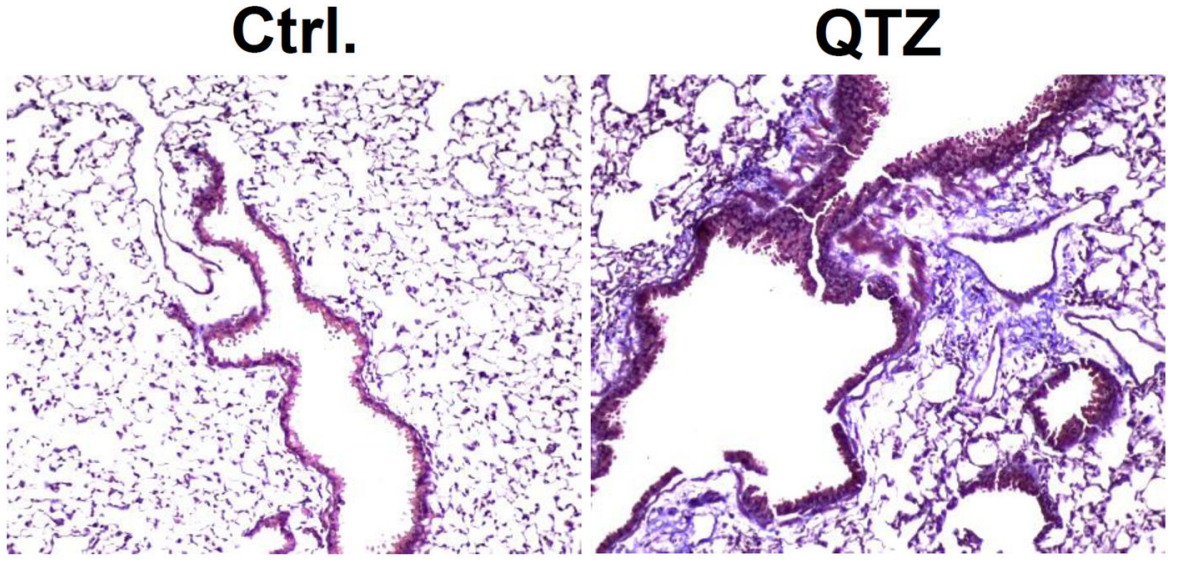

\section{Repetitive dose Single dose}

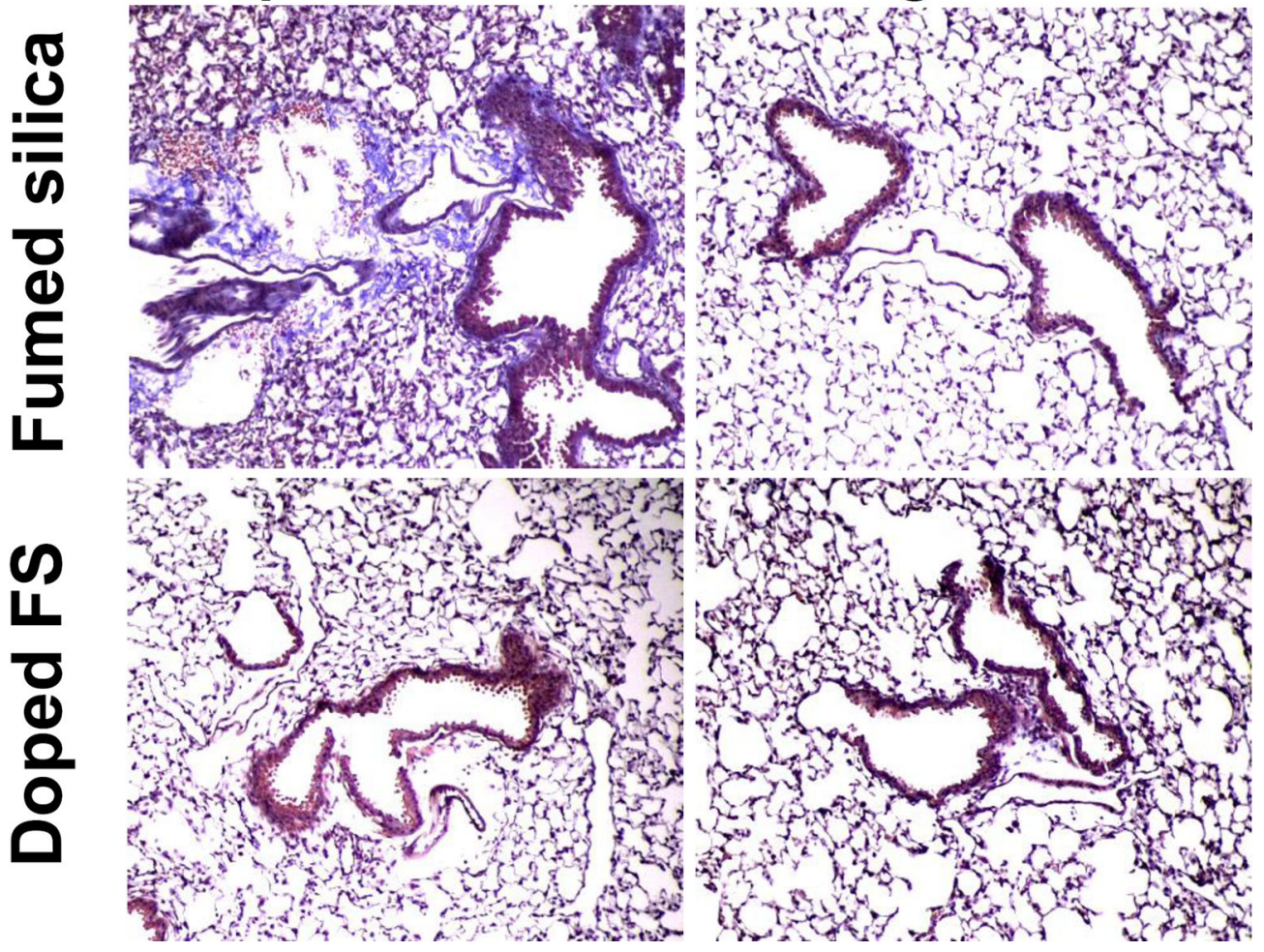

Figure 7. Ti doping ameliorated the pro-fibrogenic effects of repetitively dosed fumed silica C57BL/6 ( $\mathrm{n}=6)$ mice were exposed to either a single dose of $21 \mathrm{mg} / \mathrm{kg}$ or 3 doses of $7 \mathrm{mg} / \mathrm{kg}$ non-doped or 7\% Ti-doped fumed silica, one week apart. On day 21, BAL fluid was collected to determine (A) IL-1 $\beta$, (B) LIX, and (C) TGF- $\beta 1$ levels. (D) Total collagen content in murine lung was determined by the Sircol assay. (E) Masson's Trichrome staining was used to determine the collagen deposition in the lungs of animals treated with fumed 
silica. ${ }^{*} p<0.05$ compared to control mice. ${ }^{\#} p<0.05$ compared to pristine fumed silica treated mice. 